\title{
The effect of unitary noise on Grover's quantum search algorithm
}

\author{
Daniel Shapira, Shay Mozes, and Ofer Biham \\ Racah Institute of Physics, The Hebrew University, Jerusalem,91904,Israel
}

\begin{abstract}
The effect of unitary noise on the performance of Grover's quantum search algorithm is studied. This type of noise may result from tiny fluctuations and drift in the parameters of the (quantum) components performing the computation. The resulting operations are still unitary, but not precisely those assumed in the design of the algorithm. Here we focus on the effect of such noise in the Hadamard gate $W$, which is an essential component in each iteration of the quantum search process. To this end $W$ is replaced by a noisy Hadamard gate $U$. The parameters of $U$ at each iteration are taken from an arbitrary probability distribution (e.g. Gaussian distribution) and are characterized by their statistical moments around the parameters of $W$. For simplicity we assume that the noise is unbiased and isotropic, namely all noise variables in the parametrization we use have zero average and the same standard deviation $\epsilon$. The noise terms at different calls to $U$ are assumed to be uncorrelated. For a search space of size $N=2^{n}$ (where $n$ is the number of qubits used to span this space) it is found that as long as $\epsilon<O\left(n^{-\frac{1}{2}} N^{-\frac{1}{4}}\right)$, the algorithm maintains significant efficiency, while above this noise level its operation is hampered completely. It is also found that below this noise threshold, when the search fails, it is likely to provide a state that differs from the marked state by only a few bits. This feature can be used to search for the marked state by a classical post-processing, even if the quantum search has failed, thus improving the success rate of the search process.
\end{abstract}

PACS numbers: PACS: 03.67.Lx, 89.70.+c 


\section{INTRODUCTION}

The discovery of quantum algorithms that can solve computational problems faster than any known classical algorithm stimulated much interest in quantum information science. The known algorithms include Shor's factoring algorithm 1, 2], Grover's search algorithm [3, 4] as well as algorithms for the simulation of physical systems. One of the most serious obstacles that should be dealt with in the way to construct a quantum computer on which such algorithms can be implemented is the problem of decoherence [5]. This is the effect of the interaction between the quantum system that stores and manipulates the quantum information and the environment, that spoils the coherence of the quantum states. The effect of decoherence on Grover's search algorithm was recently studied using perturbation theory [6]. Recent progress in quantum error correcting codes [7, 8, 9, 10, 11] as well as in decoherence free sub-spaces [12, 13, 14, 15], may provide an effective way to keep the quantum states coherent and enable the implementation of useful quantum algorithms. However, a drawback of these approaches is that they involve some redundancy in the encoding of the logical quantum state, thus requiring to maintain a larger number of quantum bits in a coherent state.

The performance of a quantum computer may also be affected by unitary noise [16, 17, 18]. Such noise may result from tiny fluctuations and drift in the properties of the implemented quantum gates. These fluctuations or drifts may add stochastic perturbation elements to the Hamiltonian that describes the quantum gates that generate the unitary operations in the algorithm. The perturbated Hamiltonian is Hermitian as well, therefore the resulting operations are still unitary, although not precisely the ones assumed in the design of the algorithm. Since the implementation of a useful quantum algorithm requires a large number of one and two qubit gates, it is possible that even a tiny noise in each operation would accumulate to a considerable effect that may hamper the operation of the quantum computer.

In this paper we analyze the effect of unitary noise on Grover's quantum search algorithm. To this end we replace the Hadamard gate $W$ by a noisy (but still unitary) Hadamard gate $U$. The parameters of $U$ at each iteration are taken from an arbitrary probability distribution (e.g. Gaussian distribution) and are characterized by their statistical moments around those of $W$.

In order to simplify the calculations we assume an unbiased noise (i.e. noise with mean 
0 ) or alternately we refer to a known bias that is shifted away in every iteration of the Hadamard gate. We assume that the noise is isotropic, namely the standard deviation $\epsilon$ is the same for all the noise variables in the parameters to be defined later. This assumption is made for simplicity, and removing it does not change the main qualitative features observed in this paper.

We show that the noise reduces the success probability $P_{0}$ of the algorithm (the probability to measure the marked state, the one we are looking for, at the end of the algorithm). Consider a search problem with $N$ elements where $N=2^{n}$ ( $n$ denotes the number of qubits in the register). We find that as long as the standard deviation $\epsilon$ of the noise satisfies

$$
\epsilon \lesssim \epsilon_{0}=1.4 \frac{1}{\sqrt{n \sqrt{N}}}
$$

the algorithm maintains significant efficiency, while above this noise level, its operation is hampered completely.

We have analyzed the flow of probability out of the marked state which is caused by the noise. In the effective region of the algorithm (i.e. $\epsilon \lesssim \epsilon_{0}$ ), we find that a considerably large amount of probability diffuses from the marked state to its near neighbors, whose indices differ only in a few bits from the marked index. This result is derived analytically and verified numerically.

The "diffusive" flow from the marked state to its near neighbors can be used to enhance the efficiency of the algorithm. To this end we execute the Grover quantum search, and measure the state of the register. If it is not the marked state we classically test all its neighboring states, namely those that differ from it by only a few bits. The use of hybrid (quantum and classical) search strategies in case of unbiased and isotropic unitary noise in the Hadamard operations accomplishes a great reduction in the average searching time in comparison to the ordinary quantum search procedure that is re-execution of Grover's algorithm over and over again until the marked state is found. Moreover, hybrid strategies enable an efficient quantum search under noise levels for which the quantum search alone fails.

The paper is organized as follows: In section II we introduce the Grover quantum search algorithm. In section III we analyze the effect of unitary noisy Hadamard gates on the Grover quantum search. In section IV we present and discuss numerical results that verify 
the analytical predictions and extend the scope of discussion to noise's limits for which the analytical approximations are not valid anymore. In section $\mathrm{V}$ we examine the use of hybrid search strategies which improves the performance of the Grover quantum search with noisy Hadamard gates. Some additional details of calculations are presented in the appendix.

\section{GROVER'S SEARCH ALGORITHM}

Let $D$ be a search space containing $N$ elements. We assume, for convenience, that $N=2^{n}$, where $n$ is an integer. In this way, we may represent the elements of $D$ using an $n$-qubit register containing their indices, $i=0, \ldots, N-1$. We assume that a single marked element is the solution to the search problem. The distinction between the marked and unmarked elements can be expressed by a suitable function, $f: D \rightarrow\{0,1\}$, such that $f=1$ for the marked element, and $f=0$ for all other elements.

Suppose we wish to search the space $D$ to find the marked element, namely the element for which $f=1$. To solve this problem on a classical computer one needs to evaluate $f$ for each element, one by one, until the marked state is found. Thus, $O(N)$ evaluations of $f$ are required on a classical computer. However, if we allow the function $f$ to be evaluated coherently, there exists a sequence of unitary operations which can locate the marked element using only $O(\sqrt{N})$ queries of $f[3,4]$. This sequence of unitary operations is called Grover's quantum search algorithm.

To describe the operation of the quantum search algorithm we first introduce a register, $|\bar{x}\rangle=\left|x_{n-1}, x_{n-2}, \ldots, x_{1}, x_{0}\right\rangle$ of $n$ qubits, and an ancilla qubit, $|q\rangle$, to be used in the computation. The bar script denotes a computational string of $n$ bits, i.e. $\bar{x}=x_{n-1}, x_{n-2}, \ldots, x_{1}, x_{0}$ where the $k^{\prime}$ th bit $x_{k}$ is either one or zero, so that $|\bar{x}\rangle$ is a computational basis state vector.

We also introduce a quantum oracle, a unitary operator $\hat{O}_{f}$ which functions as a black box with the ability to recognize the solution to the search problem. We shall use the hat script for operators, namely an operator $O$ is denoted as $\hat{O}$. (For more details on how an oracle may be constructed, see Chapter 6 of Ref. [18].) The oracle performs the following unitary operation on computational basis states of the register $|\bar{x}\rangle$ and of the ancilla $|q\rangle$ :

$$
\hat{O}_{f}|\bar{x}\rangle|q\rangle=|\bar{x}\rangle|q \oplus f(\bar{x})\rangle,
$$

where $\oplus$ denotes addition modulo 2 . This definition may be uniquely extended, via linearity, 
to all states of the register and ancilla.

The oracle recognizes the marked state in the sense that if $\bar{x}$ is the marked element of the search space, $f(\bar{x})=1$, the oracle flips the ancilla qubit from $|0\rangle$ to $|1\rangle$ and vice versa, while for unmarked states the ancilla is unchanged. In Grover's algorithm the ancilla qubit is initially set to the state

$$
|q\rangle=\frac{1}{\sqrt{2}}(|0\rangle-|1\rangle) .
$$

It is easy to verify that, with this choice, the action of the oracle is:

$$
\hat{O}_{f}|\bar{x}\rangle\left(\frac{|0\rangle-|1\rangle}{\sqrt{2}}\right)=(-1)^{f(\bar{x})}|\bar{x}\rangle\left(\frac{|0\rangle-|1\rangle}{\sqrt{2}}\right) .
$$

Thus, the only effect of the oracle is to apply a phase rotation of $\pi$ radians if $\bar{x}$ is the marked state, and no phase change if $\bar{x}$ is unmarked. Since the state of the ancilla does not change, it is conventional to omit it, and write the action of the oracle as

$$
\hat{O}_{f}|\bar{x}\rangle=(-1)^{f(\bar{x})}|\bar{x}\rangle
$$

Grover's search algorithm may be summarized as follows:

1. Initialize the qubit register to $|\overline{0}\rangle=|0,0, \ldots, 0\rangle$ and the ancilla to $|0\rangle$. Then apply the gate $\hat{w} \hat{x}$ on the ancilla qubit where $\hat{x}=\left(\begin{array}{ll}0 & 1 \\ 1 & 0\end{array}\right)$ is the NOT gate and $\hat{w}=$ $\frac{1}{\sqrt{2}}\left(\begin{array}{cc}1 & 1 \\ 1 & -1\end{array}\right)$ is the Hadamard gate (The matrices are written with respect to the computational basis $|0\rangle,|1\rangle)$. The resulting state is:

$$
|0,0, \ldots, 0\rangle\left(\frac{|0\rangle-|1\rangle}{\sqrt{2}}\right)_{q}
$$

2. Grover Iterations: Repeat the following operation $T$ times:

(a) Apply the Hadamard gate on each qubit in the register.

(b) Apply the oracle, which has the effect of rotating the marked state by a phase of $\pi$ radians. Since the ancilla is always in the state $(|0\rangle-|1\rangle) / \sqrt{2}$ the effect of this operation may be described by a unitary operator acting only on the register, 
$\hat{I}_{m}=\sum_{\bar{x}}(-1)^{f(\bar{x})}|\bar{x}\rangle\langle\bar{x}|=\hat{I}-2| \bar{m}\rangle\langle\bar{m}| .\left(\hat{I}\right.$ denotes the $2^{n} \times 2^{n}$ identity operator and $|\bar{m}\rangle$ denotes the marked state, which is the one we are searching for.)

(c) Apply the Hadamard gate on each qubit in the register.

(d) Rotate the $|0,0, \ldots, 0\rangle$ state of the register by a phase of $\pi$. This rotation is similar to 2(b), except for the fact that here it is performed on a known state. It takes the form $\hat{I}_{0}=-|\overline{0}\rangle\left\langle\overline{0}\left|+\sum_{\bar{x} \neq 0}\right| \bar{x}\right\rangle\langle\bar{x}|=\hat{I}-2| \overline{0}\rangle\langle\overline{0}|$.

3. Apply the Hadamard gate on each qubit in the register and then measure the register in the computational basis.

We still need to specify the number of iterations, T. As subsequent Grover iterations are applied, the amplitude of the marked state gradually increases, while the amplitudes of the unmarked states decrease. There exists an optimal number, $T$, of iterations at which the amplitude of the marked state reaches a maximum value, and thus the probability that the measurement yields the marked state is maximal. Let us denote this probability by $P_{0}$. It has been shown [4, 19, 20] that the optimal time $T$ is:

$$
T \leq\left\lceil\frac{\pi}{4} \sqrt{N}\right\rceil
$$

where $\lceil x\rceil$ denotes the integer value of $x$. Moreover, it was shown [19] that Grover's algorithm is optimal in the sense that it is as efficient as theoretically possible [21]. For the initial state given in step 1 above, the probability to obtain a marked state at the optimal time $T$ is $P_{0}=1-O(1 / \sqrt{N})\left[19,[20]\right.$. For other initial states $P_{0}$ may be bounded away from unity 22]. Further generalizations to the original Grover's algorithm have also been suggested in [23, 24].

Following Grover's discoveries, a variety of applications were developed, in which the algorithm is used in the solution of other problems [23, 25, 26, 27, 28, 29, 30, 31, 32, 33]; Experimental implementations were also constructed using a nuclear magnetic resonance (NMR) quantum computer [34, 35] as well as on an optical device [36].

\section{ANALYSIS OF THE EFFECTS OF UNITARY NOISE}

Assuming a single marked state, the original Grover quantum search algorithm using an $n$-qubit register with $N=2^{n}$ computational basis states, could be represented by the 
operation:

$$
|g(T)\rangle \equiv \hat{G}_{0}(T)|\overline{0}\rangle=\hat{W} \hat{Q}^{T}|\overline{0}\rangle .
$$

$|g(T)\rangle$ is the quantum state after $T$ iterations which is accomplished by executing the Grover operator $\hat{G}_{0}(T)$ on the initial state $|\overline{0}\rangle=|0 \ldots 00\rangle$. When the time $T$ is optimal, the operator $\hat{G}_{0}(T)$ in Eq. (8) amplifies the absolute value of the amplitude of the marked state to 1 . The Grover operator $\hat{G}_{0}(T)$ consists of $T$ executions of Grover's iteration $\hat{Q}$ followed by a single Hadamard transform $\hat{W}$. The Grover iteration is defined as the composite operation:

$$
\hat{Q}=-\hat{I}_{0} \hat{W} \hat{I}_{m} \hat{W}
$$

where:

$$
\hat{W}=\bigotimes_{k=0}^{n-1} \hat{w}_{k} \equiv \bigotimes_{k=0}^{n-1} \frac{1}{\sqrt{2}}\left(\begin{array}{cc}
1 & 1 \\
1 & -1
\end{array}\right)_{k}
$$

is the $n$-qubit Hadamard transform $\left(\otimes\right.$ denotes a tensor product and $\bigotimes_{k=0}^{n-1} \hat{w}_{k}=\hat{w}_{n-1} \otimes$ $\left.\ldots \otimes \hat{w}_{0}\right), \hat{I}_{0}=\hat{I}-2|\overline{0}\rangle\langle\overline{0}|$ is a selective rotation by $\pi$ of the state $|\overline{0}\rangle$ and $\hat{I}_{m}=\hat{I}-2|\bar{m}\rangle\langle\bar{m}|$ is a selective rotation by $\pi$ of the marked state $|\bar{m}\rangle$ ( $(\hat{I}$ denotes the identity operator). The matrix notation used is in the computational basis representation, i.e., $|0\rangle_{k}=\left(\begin{array}{l}1 \\ 0\end{array}\right)_{k}$ and $|1\rangle_{k}=\left(\begin{array}{l}0 \\ 1\end{array}\right)_{k}$

Grover's iterations consist of the Hadamard transforms $\hat{w}_{k}, k=0, \ldots n-1$ which are one qubit gates, and the selective inversion operators that involve two qubit gates. Here we consider the effect of unitary noise in the Hadamard gates $\hat{w}_{k}, k=0, \ldots n-1$ on the performance of Grover's algorithm. To this end we define the noisy Grover iteration at time $t$ as:

$$
\hat{Q}_{t}=-\hat{I}_{0} \hat{V}_{t} \hat{I}_{m} \hat{U}_{t}
$$

Here $\hat{U}_{t}$ and $\hat{V}_{t}$ are the direct products of one qubit unitary operators, which are built of noisy executions of one qubit Hadamard gates. We assume that there are no temporal correlations in the Hadamard executions and that operations on different qubits are uncorrelated as well. 
Therefore, the operators can be written as:

$$
\begin{aligned}
\hat{U}_{t} & =\bigotimes_{k=0}^{n-1} \hat{w}_{k} e^{i \hat{a}_{k}(t)} \equiv \hat{W} e^{i \hat{A}_{t}} \\
\hat{V}_{t} & =\bigotimes_{k=0}^{n-1} e^{i \hat{c}_{k}(t)} \hat{w}_{k} \equiv e^{i \hat{C}_{t}} \hat{W}
\end{aligned}
$$

where $\hat{a}_{k}(t)$ and $\hat{c}_{k}(t)$ are one qubit stochastic operators, acting on the $k$ 'th qubit in the register at time $t$. They are Hermitian and generate the unitary noise in the one qubit Hadamard gates. Any one qubit Hermitian operator can be expanded in the basis of the Pauli operators $\hat{\sigma}_{1 k}, \hat{\sigma}_{2 k}$ and $\hat{\sigma}_{3 k}$ and the identity operator $\hat{\sigma}_{0 k}$, acting on the $k$ 'th qubit. Therefore:

$$
\begin{aligned}
\hat{a}_{k}(t) & =\sum_{\mu=0}^{3} \alpha_{\mu k}(t) \hat{\sigma}_{\mu k} \\
\hat{c}_{k}(t) & =\sum_{\mu=0}^{3} \gamma_{\mu k}(t) \hat{\sigma}_{\mu k}
\end{aligned}
$$

where:

$$
\hat{\sigma}_{0 k}=\left(\begin{array}{cc}
1 & 0 \\
0 & 1
\end{array}\right)_{k} \quad \hat{\sigma}_{1 k}=\left(\begin{array}{cc}
0 & 1 \\
1 & 0
\end{array}\right)_{k} \quad \hat{\sigma}_{2 k}=\left(\begin{array}{cc}
0 & -i \\
i & 0
\end{array}\right)_{k} \quad \hat{\sigma}_{3 k}=\left(\begin{array}{cc}
1 & 0 \\
0 & -1
\end{array}\right)_{k}
$$

are the identity and Pauli matrices in the computational basis representation and $\alpha_{\mu k}(t)$ and $\gamma_{\mu k}(t)(\mu=0,1,2,3$ and $k=1, \ldots, n)$ are real stochastic variables. Since all the $\alpha$ 's and $\gamma$ 's are produced by the same physical hardware with no correlations, their statistical properties can be expressed by their moments:

$$
\begin{aligned}
\left\langle\alpha_{\mu k}(t)\right\rangle & \equiv\left\langle\alpha_{\mu}\right\rangle \\
\left\langle\gamma_{\mu k}(t)\right\rangle & \equiv\left\langle\gamma_{\mu}\right\rangle
\end{aligned}
$$

and

$$
\begin{aligned}
\left\langle\alpha_{\mu k}(t) \alpha_{\nu k^{\prime}}\left(t^{\prime}\right)\right\rangle & \equiv \delta_{t t^{\prime}} \delta_{k k^{\prime}}\left\langle\delta \alpha_{\mu} \delta \alpha_{\nu}\right\rangle+\left\langle\alpha_{\mu}\right\rangle\left\langle\alpha_{\nu}\right\rangle \\
\left\langle\gamma_{\mu k}(t) \gamma_{\nu k^{\prime}}\left(t^{\prime}\right)\right\rangle & \equiv \delta_{t t^{\prime}} \delta_{k k^{\prime}}\left\langle\delta \gamma_{\mu} \delta \gamma_{\nu}\right\rangle+\left\langle\gamma_{\mu}\right\rangle\left\langle\gamma_{\nu}\right\rangle
\end{aligned}
$$

where:

$$
\begin{gathered}
\delta \alpha_{\mu}=\alpha_{\mu}-\left\langle\alpha_{\mu}\right\rangle \\
\delta \gamma_{\mu}=\gamma_{\mu}-\left\langle\gamma_{\mu}\right\rangle
\end{gathered}
$$


and $\delta_{t t^{\prime}}$ as well as $\delta_{k k^{\prime}}$ are Kronecker's delta functions. The noise is thus characterized by the eight real stochastic variables: $\alpha_{\mu}$ and $\gamma_{\mu}(\mu=0,1,2,3)$.

Using a series expansion of the exponentials in Eq. (12) and the identity of operators:

$$
(\hat{A} \otimes \hat{B})(\hat{C} \otimes \hat{D})=\hat{A} \hat{C} \otimes \hat{B} \hat{D}
$$

we obtain:

$$
\begin{aligned}
& \hat{A}_{t}=\sum_{k=0}^{n-1} \hat{a}_{k}(t) \\
& \hat{C}_{t}=\sum_{k=0}^{n-1} \hat{c}_{k}(t) .
\end{aligned}
$$

The operators $\hat{A}_{t}$ and $\hat{C}_{t}$ are built as sums of $n$ one qubit operators. Therefore, the only non-zero elements in their $2^{n} \times 2^{n}$ matrix representation in the computational basis are those having indices that differ in no more than one bit. Denoting two computational basis states: $|\bar{x}\rangle=\left|x_{n-1}, x_{n-2}, \ldots x_{0}\right\rangle$ and $|\bar{y}\rangle=\left|y_{n-1}, y_{n-2}, \ldots y_{0}\right\rangle\left(x_{k}=0,1\right.$ and $y_{k}=0,1$ where $k=0, \ldots, n-1)$, the following matrix elements can be calculated using Eqs. (13)-(14) and (18):

$$
\left\langle\bar{x}\left|\hat{A}_{t}\right| \bar{y}\right\rangle=\left\{\begin{array}{cc}
\sum_{k=0}^{n-1}\left\{\alpha_{0 k}(t)+(-1)^{y_{k}} \alpha_{3 k}(t)\right\} & \text { if }\|\bar{x}-\bar{y}\|=0, \\
\alpha_{1 k}(t)+i(-1)^{y_{k}} \alpha_{2 k}(t) & \text { if }\|\bar{x}-\bar{y}\|=1\left(x_{k}=\neg y_{k}\right), \\
0 & \text { if }\|\bar{x}-\bar{y}\|>1
\end{array}\right.
$$

and

$$
\left\langle\bar{x}\left|\hat{C}_{t}\right| \bar{y}\right\rangle=\left\{\begin{array}{cc}
\sum_{k=0}^{n-1}\left\{\gamma_{0 k}(t)+(-1)^{y_{k}} \gamma_{3 k}(t)\right\} & \text { if }\|\bar{x}-\bar{y}\|=0, \\
\gamma_{1 k}(t)+i(-1)^{y_{k}} \gamma_{2 k}(t) & \text { if }\|\bar{x}-\bar{y}\|=1\left(x_{k}=\neg y_{k}\right), \\
0 & \text { if }\|\bar{x}-\bar{y}\|>1 .
\end{array}\right.
$$

The norm $\|\bar{x}-\bar{y}\|$ is the Hamming distance that counts the number of bits that are different in $\bar{x}$ and $\bar{y}$. Similarly, one can write:

$$
\begin{aligned}
\hat{W} \hat{A}_{t} \hat{W} & =\sum_{k=0}^{n-1} \hat{w}_{k} \hat{a}_{k}(t) \hat{w}_{k} \\
\hat{W} \hat{C}_{t} \hat{W} & =\sum_{k=0}^{n-1} \hat{w}_{k} \hat{c}_{k}(t) \hat{w}_{k}
\end{aligned}
$$

so that:

$$
\left\langle\bar{x}\left|\hat{W} \hat{A}_{t} \hat{W}\right| \bar{y}\right\rangle=\left\{\begin{array}{cc}
\sum_{k=0}^{n-1}\left\{\alpha_{0 k}(t)+(-1)^{y_{k}} \alpha_{1 k}(t)\right\} & \text { if }\|\bar{x}-\bar{y}\|=0, \\
\alpha_{3 k}(t)-i(-1)^{y_{k}} \alpha_{2 k}(t) & \text { if }\|\bar{x}-\bar{y}\|=1\left(x_{k}=\neg y_{k}\right), \\
0 & \text { if }\|\bar{x}-\bar{y}\|>1
\end{array}\right.
$$


and

$$
\left\langle\bar{x}\left|\hat{W} \hat{C}_{t} \hat{W}\right| \bar{y}\right\rangle=\left\{\begin{array}{cc}
\sum_{k=0}^{n-1}\left\{\gamma_{0 k}(t)+(-1)^{y_{k}} \gamma_{1 k}(t)\right\} & \text { if }\|\bar{x}-\bar{y}\|=0, \\
\gamma_{3 k}(t)-i(-1)^{y_{k}} \gamma_{2 k}(t) & \text { if }\|\bar{x}-\bar{y}\|=1\left(x_{k}=\neg y_{k}\right), \\
0 & \text { if }\|\bar{x}-\bar{y}\|>1
\end{array}\right.
$$

are elements of $2^{n} \times 2^{n}$ sparse matrices as well.

The noisy Hadamard transforms $\hat{U}_{t}$ and $\hat{V}_{t}$ defined in Eq. (12) can now be written explicitly into the noisy Grover's iteration (11) for any time $t$ :

$$
\begin{aligned}
\hat{Q}_{t} & =-\hat{I}_{0} e^{i \hat{C}_{t}} \hat{W} \hat{I}_{m} \hat{W} e^{i \hat{A}_{t}} \\
& =\left(\hat{I}_{0} e^{i \hat{C}_{t}} \hat{I}_{0}\right)\left(-\hat{I}_{0} \hat{W} \hat{I}_{m} \hat{W}\right) e^{i \hat{A}_{t}} \\
& =\hat{I}_{0} e^{i \hat{C}_{t}} \hat{I_{0}} \hat{Q} e^{i \hat{A}_{t}}=e^{i \hat{I}_{0} \hat{C}_{t} \hat{I}_{0}} \hat{Q} e^{i \hat{A}_{t}}
\end{aligned}
$$

and the entire search process after $T$ iterations is then given by:

$$
\begin{aligned}
\hat{G}(T) & =\hat{U}_{T+1} \hat{Q}_{T} \ldots \hat{Q}_{1} \\
& =\left(\hat{W} e^{i \hat{A}_{T+1}}\right)\left(e^{i \hat{I}_{0} \hat{C}_{T} \hat{I}_{0}} \hat{Q} e^{i \hat{A}_{T}}\right)\left(e^{i \hat{I}_{0} \hat{C}_{T-1} \hat{I}_{0}} \ldots e^{i \hat{A}_{2}}\right)\left(e^{i \hat{I}_{0} \hat{C}_{1} \hat{I}_{0}} \hat{Q} e^{i \hat{A}_{1}}\right) .
\end{aligned}
$$

A Taylor series expansion now yields

$$
\begin{aligned}
e^{i \hat{A}_{t+1}} e^{i \hat{I}_{0} \hat{C}_{t} \hat{I}_{0}} & \approx\left(\hat{I}+i \hat{A}_{t+1}-\frac{1}{2} \hat{A}_{t+1}^{2}+\ldots\right)\left(\hat{I}+i \hat{I}_{0} \hat{C}_{t} \hat{I}_{0}-\frac{1}{2} \hat{I}_{0} \hat{C}_{t}^{2} \hat{I}_{0}+\ldots\right) \\
& \approx \hat{I}+i \hat{E}_{t}^{(1)}-\frac{1}{2} \hat{E}_{t}^{(2)}+\ldots
\end{aligned}
$$

where

$$
\begin{aligned}
& \hat{E}_{t}^{(1)}=\hat{A}_{t+1}+\hat{I}_{0} \hat{C}_{t} \hat{I}_{0} \\
& \hat{E}_{t}^{(2)}=\hat{A}_{t+1}^{2}+2 \hat{A}_{t+1} \hat{I}_{0} \hat{C}_{t} \hat{I}_{0}+\hat{I}_{0} \hat{C}_{t}^{2} \hat{I}_{0}
\end{aligned}
$$

are the first and the second order deviations, caused by the noise, so that:

$$
\begin{aligned}
\hat{G}(T) & \approx \hat{W}\left(\hat{I}+i \hat{E}_{T}^{(1)}-\frac{1}{2} \hat{E}_{T}^{(2)}+\ldots\right) \hat{Q}\left(\hat{I}+i \hat{E}_{T-1}^{(1)}-\frac{1}{2} \hat{E}_{T-1}^{(2)}+\ldots\right) \ldots \hat{Q}\left(\hat{I}+i \hat{E}_{0}^{(1)}-\frac{1}{2} \hat{E}_{0}^{(2)}+\ldots\right) \\
& =\hat{G}_{0}(T)+\hat{G}_{1}(T)+\hat{G}_{2}(T)+\ldots
\end{aligned}
$$

where we define $\hat{C}_{0} \equiv 0$. The operator

$$
\hat{G}_{0}(T)=\hat{W} \hat{Q}^{T}
$$


is the original Grover quantum search operator (without noise), while $\hat{G}_{l}(T)$ are the components of perturbation of order $l(l=1,2 \ldots)$. Specifically, the leading terms of the perturbation satisfy:

$$
\hat{G}_{1}(T)=i \hat{W}\left\{\sum_{t=0}^{T}\left(\hat{Q}^{T-t} \hat{E}_{t}^{(1)} \hat{Q}^{t}\right)\right\}
$$

and

$$
\hat{G}_{2}(T)=-\frac{1}{2} \hat{W}\left\{\sum_{t=0}^{T}\left(\hat{Q}^{T-t} \hat{E}_{t}^{(2)} \hat{Q}^{t}\right)+2 \sum_{t=1}^{T} \sum_{t^{\prime}=0}^{t-1}\left(\hat{Q}^{T-t} \hat{E}_{t}^{(1)} \hat{Q}^{t-t^{\prime}} \hat{E}_{t^{\prime}}^{(1)} \hat{Q}^{t^{\prime}}\right)\right\} .
$$

Those additional perturbation components consist of summations over time indices $t$ of operator multiplications. These multiplications include the noise generators $\hat{A}_{t}$ and $\hat{C}_{t}$ where the number of their appearances determines the order of the perturbation. They also include non-stochastic operators: powers of the original Grover iteration $\hat{Q}$ and selective inversions around the initial state of zeros $\hat{I}_{0}$. In order to understand the behavior of the operator $\hat{G}(T)$, let us first focus on the non-stochastic operators which appear in those multiplications.

For any arbitrary state $|\psi\rangle$ of the $n$-qubit register

$$
\begin{aligned}
\hat{Q}|\psi\rangle & =-\hat{I}_{0} \hat{W} \hat{I}_{m} \hat{W}|\psi\rangle \\
& =-(\hat{I}-2|\overline{0}\rangle\langle\overline{0}|) \hat{W}(\hat{I}-2|\bar{m}\rangle\langle\bar{m}|) \hat{W}|\psi\rangle \\
& =-|\psi\rangle+2\{\langle\overline{0} \mid \psi\rangle-2\langle\overline{0}|\hat{W}| \bar{m}\rangle\langle\bar{m}|\hat{W}| \psi\rangle\}|\overline{0}\rangle+2\langle\bar{m}|\hat{W}| \psi\rangle \hat{W}|\bar{m}\rangle .
\end{aligned}
$$

In particular:

$$
\hat{Q}|\overline{0}\rangle=\left\{1-4|\langle\bar{m}|\hat{W}| \overline{0}\rangle|^{2}\right\}|\overline{0}\rangle+2\langle\bar{m}|\hat{W}| \overline{0}\rangle \hat{W}|\bar{m}\rangle
$$

and

$$
\hat{Q} \hat{W}|\bar{m}\rangle=-2\langle\overline{0}|\hat{W}| \bar{m}\rangle|\overline{0}\rangle+\hat{W}|\bar{m}\rangle
$$

The operator $\hat{Q}$ acts as a linear transformation within a 3-dimensional vector space that is spanned by the three vectors: $|\psi\rangle,|\overline{0}\rangle$ and $\hat{W}|\bar{m}\rangle$. In case that the state vector $|\psi\rangle$ is linearly independent on the vectors $|\overline{0}\rangle$ and $\hat{W}|\bar{m}\rangle$, the following representation of vectors basis can be performed:

$$
|\psi\rangle \leftrightarrow\left(\begin{array}{c}
1 \\
0 \\
0
\end{array}\right) \quad|\overline{0}\rangle \leftrightarrow\left(\begin{array}{c}
0 \\
1 \\
0
\end{array}\right) \quad \hat{W}|\bar{m}\rangle \leftrightarrow\left(\begin{array}{l}
0 \\
0 \\
1
\end{array}\right)
$$

Note that the these vectors are not represented in the computational basis. However, they are linearly independent and hence can be treated as a standard basis in a simple 
3-dimensional vector space in which an inner product is not defined. Namely, the above representation does not imply orthogonality of the vectors $|\psi\rangle,|\overline{0}\rangle$ and $\hat{W}|\bar{m}\rangle$, and the following matrix calculations are exact and involve no approximations at all. This representation does not change eigenvalues and eigenvectors from those obtained for an orthonormal basis.

Now, denoting $\langle\overline{0}|\hat{W}| \bar{m}\rangle=\langle\bar{m}|\hat{W}| \overline{0}\rangle=\frac{1}{\sqrt{N}}$, the operator $\hat{Q}$, which is a regular linear transformation acting on a simple 3-dimensional vector space, is represented by the 3-dimensional matrix:

where:

$$
\hat{Q}=\left(\begin{array}{ccc}
-1 & 0 & 0 \\
q_{1} & 1-\frac{4}{N} & -\frac{2}{\sqrt{N}} \\
q_{2} & \frac{2}{\sqrt{N}} & 1
\end{array}\right)
$$

$$
\begin{aligned}
q_{1} & =2\left\{\langle\overline{0} \mid \psi\rangle-\frac{2}{\sqrt{N}}\langle\bar{m}|\hat{W}| \psi\rangle\right\} \\
q_{2} & =2\langle\bar{m}|\hat{W}| \psi\rangle .
\end{aligned}
$$

Powers of the operator $\hat{Q}$ do not exceed from the 3-dimensional sub-space spanned by $|\psi\rangle$, $|\overline{0}\rangle$ and $\hat{W}|\bar{m}\rangle$. Diagonalizing the matrix $\hat{Q}$ we obtain

$$
\hat{Q}_{D}=\left(\begin{array}{ccc}
\lambda_{0} & 0 & 0 \\
0 & \lambda_{+} & 0 \\
0 & 0 & \lambda_{-}
\end{array}\right)
$$

where the eigenvalues are $\lambda_{0}=-1$ and $\lambda_{ \pm}=e^{ \pm i \omega}$, and $\omega$ is given by:

$$
\cos \omega=1-\frac{2}{N}
$$

The diagonalizing matrix whose columns consist of the three eigenvectors takes the form:

$$
\hat{X}=\left(\begin{array}{ccc}
1 & 0 & 0 \\
v_{1} & 1 & 1 \\
v_{2} & e^{+i \varphi} & e^{-i \varphi}
\end{array}\right)
$$

where

$$
e^{ \pm i \varphi}=\frac{-\frac{2}{\sqrt{N}}}{1-e^{ \pm i \omega}}
$$

and

$$
\begin{aligned}
v_{1} & =\frac{\frac{1}{\sqrt{N}}\langle\bar{m}|\hat{W}| \psi\rangle-\langle\overline{0} \mid \psi\rangle}{1-\frac{1}{N}} \\
v_{2} & =\frac{\frac{1}{\sqrt{N}}\langle\overline{0} \mid \psi\rangle-\langle\bar{m}|\hat{W}| \psi\rangle}{1-\frac{1}{N}} .
\end{aligned}
$$


We now obtain an explicit expression for powers of $\hat{Q}$

$$
\begin{aligned}
\hat{Q}^{t} & =\hat{X} \hat{Q}_{D}^{t} \hat{X}^{-1} \\
& =\left(\begin{array}{ccc}
(-1)^{t} & 0 & 0 \\
v_{1}\left[(-1)^{t}-k(t)\right]+v_{2} l(t) & k(t) & -l(t) \\
v_{2}\left[(-1)^{t}-m(t)\right]-v_{1} l(t) & l(t) & m(t)
\end{array}\right)
\end{aligned}
$$

where:

$$
\begin{aligned}
k(t) & =-\frac{\sin (\omega t-\varphi)}{\sin (\varphi)} \\
l(t) & =-\frac{\sin (\omega t)}{\sin (\varphi)} \\
m(t) & =\frac{\sin (\omega t+\varphi)}{\sin (\varphi)} .
\end{aligned}
$$

The expressions above can be simplified in the limit of large $N$. In this limit the frequency $\omega$ given by Eq. (39) can be approximated by

$$
\omega=\frac{2}{\sqrt{N}}+O\left(\frac{1}{N^{\frac{3}{2}}}\right) .
$$

Under this approximation Eq. (41) becomes

$$
e^{ \pm i \varphi}=\mp i+O\left(\frac{1}{\sqrt{N}}\right)
$$

namely

$$
\varphi=-\frac{\pi}{2}+O\left(\frac{1}{\sqrt{N}}\right)
$$

As a result:

$$
k(t) \approx m(t)=\cos (\omega t)+O\left(\frac{1}{\sqrt{N}}\right)
$$

and

$$
l(t)=\sin (\omega t)+O\left(\frac{1}{\sqrt{N}}\right) .
$$

Moreover, as we shall see later, the superposition $|\psi\rangle$ appears in our first order calculation in only two forms. Either $|\psi\rangle=|\bar{y}\rangle$ where $|\bar{y}\rangle$ is a computational basis state perpendicular to $|\overline{0}\rangle$ or $|\psi\rangle=\hat{W}|\bar{y}\rangle$ where $|\bar{y}\rangle$ is a computational basis state perpendicular to the marked state $|\bar{m}\rangle$. In the first case we obtain

$$
\begin{gathered}
v_{1}=\frac{\frac{1}{\sqrt{N}}\langle\bar{m}|\hat{W}| \bar{y}\rangle}{1-\frac{1}{N}}=O\left(\frac{1}{N}\right) \\
v_{2}=\frac{-\langle\bar{m}|\hat{W}| \bar{y}\rangle}{1-\frac{1}{N}}=O\left(\frac{1}{\sqrt{N}}\right) .
\end{gathered}
$$


In the second case we find that

$$
\begin{gathered}
v_{1}=\frac{-\langle\overline{0}|\hat{W}| \bar{y}\rangle}{1-\frac{1}{N}}=O\left(\frac{1}{\sqrt{N}}\right) \\
v_{2}=\frac{\frac{1}{\sqrt{N}}\langle\overline{0}|\hat{W}| \bar{y}\rangle}{1-\frac{1}{N}}=O\left(\frac{1}{N}\right) .
\end{gathered}
$$

Thus in both cases the matrix of Eq. (43) takes the form:

$$
\hat{Q}^{t}=\left(\begin{array}{ccc}
(-1)^{t} & 0 & 0 \\
O\left(\frac{1}{\sqrt{N}}\right) & \cos (\omega t) & -\sin (\omega t) \\
O\left(\frac{1}{\sqrt{N}}\right) & \sin (\omega t) & \cos (\omega t)
\end{array}\right)
$$

This means that $\hat{Q}^{t}$ acts simultaneously, up to $O\left(\frac{1}{\sqrt{N}}\right)$, as a 2-dimensional rotator in the sub-space spanned by the state vectors $|\overline{0}\rangle$ and $\hat{W}|\bar{m}\rangle$, as well as selective phase invertor (according to the parity of the power $t$ ) of any state vector $|\psi\rangle$ that is independent on $|\overline{0}\rangle$ and $\hat{W}|\bar{m}\rangle$.

The selective inversion around $|\overline{0}\rangle$ (denoted as $\hat{I}_{0}$ ) also preserves the 3-dimensional simple vector space spanned by $|\psi\rangle$, For any superposition $|\psi\rangle$

$$
\hat{I}_{0}|\psi\rangle=(\hat{I}-2|\overline{0}\rangle\langle\overline{0}|)|\psi\rangle=|\psi\rangle-2\langle\overline{0} \mid \psi\rangle|\overline{0}\rangle,
$$

where $\langle\overline{0} \mid \psi\rangle=0$ for the case of $|\psi\rangle=|\bar{y}\rangle \neq|\overline{0}\rangle$ and $\langle\overline{0} \mid \psi\rangle=\frac{1}{\sqrt{N}}$ for the case of $|\psi\rangle=\hat{W}|\bar{y}\rangle$ $(|\bar{y}\rangle \neq|\bar{m}\rangle)$, where $|\bar{y}\rangle$ denotes an arbitrary computational basis state. Particularly we can write:

$$
\hat{I}_{0}|\overline{0}\rangle=-|\overline{0}\rangle
$$

and

$$
\hat{I}_{0} \hat{W}|\bar{m}\rangle=\hat{W}|\bar{m}\rangle-\frac{2}{\sqrt{N}}|\overline{0}\rangle
$$

Hence in both cases the selective inversion can be represented by:

$$
\hat{I}_{0}=\left(\begin{array}{ccc}
1 & 0 & 0 \\
O\left(\frac{1}{\sqrt{N}}\right) & -1 & O\left(\frac{1}{\sqrt{N}}\right) \\
0 & 0 & 1
\end{array}\right) \text {. }
$$

The effect of the stochastic operators $\hat{A}_{t}$ and $\hat{C}_{t}$, given by Eqs. (19) - (23) on a compu- 
tational basis state $|\bar{y}\rangle$ is given by:

$$
\begin{aligned}
\hat{A}_{t}|\bar{y}\rangle & =\left\langle\bar{y}\left|\hat{A}_{t}\right| \bar{y}\right\rangle|\bar{y}\rangle+\sum_{k=0}^{n-1}\left\langle\bar{y}_{1 k}\left|\hat{A}_{t}\right| \bar{y}\right\rangle\left|\bar{y}_{1 k}\right\rangle \\
\hat{A}_{t} \hat{W}|\bar{y}\rangle & =\left\langle\bar{y}\left|\hat{W} \hat{A}_{t} \hat{W}\right| \bar{y}\right\rangle \hat{W}|\bar{y}\rangle+\sum_{k=0}^{n-1}\left\langle\bar{y}_{1 k}\left|\hat{W} \hat{A}_{t} \hat{W}\right| \bar{y}\right\rangle \hat{W}\left|\bar{y}_{1 k}\right\rangle \\
\hat{C}_{t}|\bar{y}\rangle & =\left\langle\bar{y}\left|\hat{C}_{t}\right| \bar{y}\right\rangle|\bar{y}\rangle+\sum_{k=0}^{n-1}\left\langle\bar{y}_{1 k}\left|\hat{C}_{t}\right| \bar{y}\right\rangle\left|\bar{y}_{1 k}\right\rangle \\
\hat{C}_{t} \hat{W}|\bar{y}\rangle & =\left\langle\bar{y}\left|\hat{W} \hat{C}_{t} \hat{W}\right| \bar{y}\right\rangle \hat{W}|\bar{y}\rangle+\sum_{k=0}^{n-1}\left\langle\bar{y}_{1 k}\left|\hat{W} \hat{C}_{t} \hat{W}\right| \bar{y}\right\rangle \hat{W}\left|\bar{y}_{1 k}\right\rangle .
\end{aligned}
$$

Here $\left\{\left|\bar{y}_{1 k}\right\rangle\right\}_{0 \leq k \leq n-1}$ are the $n$ basis vectors, that are different from the basis vector $|\bar{y}\rangle$ in a single qubit only, denoted as the $k$ 'th qubit in the register. For example, in a 5 qubit system, where $|\bar{y}\rangle=|\overline{17}\rangle=|10001\rangle,\left\{\left|\bar{y}_{1 k}\right\rangle\right\}_{0 \leq k \leq 4}$ is the set of computational basis states which includes $|10000\rangle,|10011\rangle,|10101\rangle$, |11001 $\rangle$ and $|00000\rangle$. Any operation of either $\hat{A}_{t}$ or $\hat{C}_{t}$ on basis state vectors of the form $|\bar{y}\rangle$ or $\hat{W}|\bar{y}\rangle$, increases the dimension of the relevant vectors space by $n$ additional independent directions determined by $\left\{\left|\bar{y}_{1 k}\right\rangle\right\}_{0 \leq k \leq n-1}$ and $\left\{\hat{W}\left|\bar{y}_{1 k}\right\rangle\right\}_{0 \leq k \leq n-1}$ respectively.

Grover's search algorithm is performed by executing the operator $\hat{G}(T)$ on the initial state of zeros $|\overline{0}\rangle$. Ignoring the effect of the unitary noise in the Hadamard transforms (i.e. considering $\hat{G}(T) \approx \hat{G}_{0}(T)=\hat{W} \hat{Q}^{T}$ ), one finds that Grover's output state vector after time $T$ lays in a 2-dimensional sub-space, spanned by the Hadamard operation on the initial state of zeros $\hat{W}|\overline{0}\rangle$ and the marked state $|\bar{m}\rangle$ (Note that $\hat{Q}^{T}$ rotates the state vector $|\overline{0}\rangle$ in the 2-dimensional sub-space spanned by $|\overline{0}\rangle$ and $\hat{W}|\bar{m}\rangle$ while $\hat{W}^{2}=\hat{I}$ is the identity operator).

The unitary noise in the Hadamard transforms add perturbation elements to Grover's operator $\hat{G}_{0}(T)$ [see Eq. (28)]. These elements consist of the noise generators $\hat{A}_{t}$ and $\hat{C}_{t}$, powers of the Grover iteration $\hat{Q}$ and selective inversion $\hat{I}_{0}$. Executions of $\hat{Q}$ and $\hat{I}_{0}$ do not remain confined to a certain 3-dimensional sub-space. However, any operation of either $\hat{A}_{t}$ or $\hat{C}_{t}$ on a basis state vector of the form $|\bar{y}\rangle$ or $\hat{W}|\bar{y}\rangle,\left(|\bar{y}\rangle=\left|y_{n-1}, y_{n-2}, \ldots y_{0}\right\rangle\right.$ where $\left.y_{k}=0,1\right)$, extends the superposition by $n$ additional independent state vectors. The higher the order $l$ of the perturbation component $\hat{G}_{l}(T)$ [see Eq. (25)], the larger the number of executions of the noise generators.

Consider a realization of the noise in which all the $\alpha$ 's and the $\gamma$ 's are of order $\epsilon$. Table【 shows the vectors which appear in the superpositions produced by the leading perturbation 
components of Grover's expansion (28) with their corresponding order of the noise. An optimal time $T=T_{0}$, in which the noiseless Grover's operator returns exactly the marked state $|\bar{m}\rangle$ is assumed (i.e. $\hat{G}_{0}\left(T_{0}\right)|\overline{0}\rangle=|\bar{m}\rangle$ ). The vectors $\left\{\left|\bar{m}_{1 k}\right\rangle\right\}_{0 \leq k \leq n-1}$ and $\left\{\left|\overline{0}_{1 k}\right\rangle\right\}_{0 \leq k \leq n-1}$ are the $n$ vectors that are respectively different from the marked state $|\bar{m}\rangle$ and the state of zeros $|\overline{0}\rangle$ in their $k$ 'th bit only, the vectors $\left\{\left|\bar{m}_{2 k_{1} k_{2}}\right\rangle\right\}_{0 \leq k_{1}<k_{2} \leq n-1}$ and $\left\{\left|\overline{0}_{2 k_{1} k_{2}}\right\rangle\right\}_{0 \leq k_{1}<k_{2} \leq n-1}$ denote the $C_{2}^{n}$ vectors that are respectively different from those vectors in the $k_{1}$ 'th and $k_{2}$ 'th bits and so on ( $C_{l}^{n}$ is the binomial coefficient $\left.C_{l}^{n}=\frac{n !}{l !(n-l) !}\right)$.

Given the marked state $|\bar{m}\rangle$ we define a neighborhood class $l$ which includes all computational basis states $|\bar{x}\rangle$ that are different from the marked state $|\bar{m}\rangle$ in exactly $l$ bits. e.g. consider a 5 qubit system with marked state $|\bar{m}\rangle=|\overline{17}\rangle=|10001\rangle$, one finds the state $|\bar{x}\rangle=|\overline{3} 1\rangle=|11111\rangle$ in the 3'rd neighborhood class.

Table【clearly shows that in spite of $T$ being the optimal time $T_{0}$, the noise causes a flow of probability from the marked state $|\bar{m}\rangle$. (In case that $T$ is not optimal, the probability flows out of the original 2-dimensional Grover's space, spanned by $|\bar{m}\rangle$ and $\hat{W}|\overline{0}\rangle$.)

A certain portion of the probability "diffuses" from the marked state $|\bar{m}\rangle$ to its neighbors $\left\{\left|\bar{m}_{1 k}\right\rangle\right\}_{0 \leq k \leq n-1},\left\{\left|\bar{m}_{2 k_{1} k_{2}}\right\rangle\right\}_{0 \leq k_{1}<k_{2} \leq n-1}$ etc., where any perturbation component of order $l$ $\left(\hat{G}_{l}(T)\right)$ contributes amplitudes of order $O\left(\epsilon^{l}\right)$ to all the states within neighborhood classes of order $l^{\prime} \leq l$.

Another portion of probability involves the Hadamard operator and hence flows uniformly to all $N$ computational basis states. Since the first component which includes a Hadamard operator is $O\left(\epsilon^{1}\right)$, the contribution of this flow to each computational basis state is $O\left(\frac{\epsilon}{\sqrt{N}}\right)$.

Clearly in the limit of large $N$, the "diffusive" flow into states of small $l$ neighborhood class (of order $O\left(\epsilon^{l}\right)$ ) is much stronger than the uniform flow (of order $O\left(\frac{\epsilon}{\sqrt{N}}\right)$ ). We call these states $|\bar{m}\rangle$ 's near states. All other states are considered far states. There is a trade-off; on one hand the probability to measure a certain near state is larger than the probability to measure a certain far one. On the other hand there are much more far states $(O(N)$ far states where $\left.N=2^{n}\right)$ than near states $\left(O\left(n^{l}\right)\right.$ near states, where $l$ is small).

We will now quantify the performance of Grover's search algorithm in the presence of unitary noise. To this end we will calculate the average probabilities to measure certain basis states $|\bar{x}\rangle=\left|x_{n-1}, x_{n-2}, \ldots x_{0}\right\rangle$ at the optimal measurement time. Without noise, at the optimal measurement time the probability to measure the marked state is $p_{0}=1$. Thus, the probability of any other state is zero. According to Eq. (8) using the matrix 
representation in Eq. (50), the unperturbed probability of measuring an unmarked basis state $|\bar{x}\rangle($ i.e. $|\bar{x}\rangle \neq|\bar{m}\rangle)$ at a certain time $T$ is:

$$
p_{0}(\bar{x}, T)=\left|\left\langle\bar{x}\left|\hat{G}_{0}(T)\right| \overline{0}\right\rangle\right|^{2}=\left|\frac{1}{\sqrt{N}} \cos (\omega T)+O\left(\frac{1}{N}\right)\right|^{2}
$$

where $N=2^{n}$ is the total number of computational basis states. Therefore the optimal measurement time is given by:

$$
T_{0}=\frac{\pi}{2 \omega}+\frac{O\left(\frac{1}{\sqrt{N}}\right)}{\omega}=\frac{\pi}{4} \sqrt{N}+O(1) .
$$

Adding a perturbation to Grover's quantum search operator, a second order expansion of the probability to measure a state $|\bar{x}\rangle$ at time $T$ gives:

$$
p(\bar{x}, T)=|\langle\bar{x}|\hat{G}(T)| \overline{0}\rangle|^{2}=\left|\left\langle\bar{x}\left|\hat{G}_{0}(T)\right| \overline{0}\right\rangle\right|^{2}+\Delta p(\bar{x}, T)
$$

where:

$$
\Delta p(\bar{x}, T)=R(\bar{x}, T)+\left|\left\langle\bar{x}\left|\hat{G}_{1}(T)\right| \overline{0}\right\rangle\right|^{2}
$$

and

$$
R(\bar{x}, T)=2\left\langle\bar{x}\left|\hat{G}_{0}(T)\right| \overline{0}\right\rangle \operatorname{Re}\left\{\left\langle\bar{x}\left|\hat{G}_{1}(T)\right| \overline{0}\right\rangle+\left\langle\bar{x}\left|\hat{G}_{2}(T)\right| \overline{0}\right\rangle\right\}
$$

The normalization condition dictates that the probability to measure the marked state $|\bar{m}\rangle$ at any time $T$ satisfies $p(\bar{m}, T)=1-\sum_{\bar{x} \neq \bar{m}} p(\bar{x}, T)$. When $T$ is around the optimal time $T_{0}$, the probability to measure the marked state approaches unity. Therefore, all the terms that are much less than $O\left(\frac{1}{N}\right)$ and appear in the $N-1$ probabilities $p(\bar{x}, T)$ to measure unmarked states $|\bar{x}\rangle$ respectively, become negligible.

For time $T$ close to $T_{0}$ an expression of the form $T=T_{0}+t$ where $|t|=O(1) \ll T_{0}=$ $O(\sqrt{N})$ can be written. Hence, for any unmarked state the estimation:

$$
\left|\left\langle\bar{x}\left|\hat{G}_{0}(T)\right| \overline{0}\right\rangle\right|=\frac{1}{\sqrt{N}}|\cos (\omega T)|=\frac{1}{\sqrt{N}}|\sin (\omega t)|=\frac{\omega}{\sqrt{N}}|t|=O\left(\frac{1}{N}\right)
$$

can be made. Therefore, $\left|\left\langle\bar{x}\left|\hat{G}_{0}(T)\right| \overline{0}\right\rangle\right|^{2}=O\left(\frac{1}{N^{2}}\right) \ll O\left(\frac{1}{N}\right)$ is negligible.

On the other hand, a small perturbation around the original Grover operator $\hat{G}_{0}(T)$ is assumed. Namely, $\left|\left\langle\bar{x}\left|\hat{G}_{2}(T)\right| \overline{0}\right\rangle\right| \ll\left|\left\langle\bar{x}\left|\hat{G}_{1}(T)\right| \overline{0}\right\rangle\right| \ll O(1)$, so that $|R(\bar{x}, T)| \ll O\left(\frac{1}{N}\right)$ and is therefore negligible. 
Hence, the mean probability of measuring a computational basis state $|\bar{x}\rangle$ at time $T$ around the optimal time $T_{0}$ is given by:

$$
\langle p(\bar{x}, T)\rangle \approx \begin{cases}\left\langle\left|\left\langle\bar{x}\left|\hat{G}_{1}(T)\right| \overline{0}\right\rangle\right|^{2}\right\rangle & \text { if } \bar{x} \neq \bar{m}, \\ 1-\sum_{\bar{x} \neq \bar{m}}\left\langle\left(\left|\left\langle\bar{x}\left|\hat{G}_{1}(T)\right| \overline{0}\right\rangle\right|^{2}\right)\right\rangle & \text { if } \bar{x}=\bar{m}\end{cases}
$$

where $|\bar{m}\rangle$ is the marked state, \langle\rangle denotes the averaging on the noise and

$$
\left\langle\bar{x}\left|\hat{G}_{1}(T)\right| \overline{0}\right\rangle=i\left\{\sum_{t=0}^{T}\left\langle\bar{x}\left|\hat{W} \hat{Q}^{T-t}\left(\hat{A}_{t+1}+\hat{I}_{0} \hat{C}_{t} \hat{I}_{0}\right) \hat{Q}^{t}\right| \overline{0}\right\rangle\right\}
$$

using the definition of $\hat{C}_{t=0} \equiv 0$.

In the Appendix, we apply the matrix representations which appear in Eqs. (501) and (54) as well as the noise matrix elements in Eqs. (19)-(201) and (22)-(23) to calculate the leading terms of the mean measurement probabilities at the optimal measurement time in the limit of weak noise and large $N$. We assume that the two Hadamard transforms, which appear in each Grover iteration, are implemented with similar hardware. We also assume that the noise is unbiased, or alternately we refer to the case in which the exact values of the bias elements are known (a-priory) and can be shifted away in every iteration of the Hadamard gate. For further simplification we only consider an isotropic noise. Thus, statistical moments of the noise defined in Eqs. (15) and (16) takes the form:

$$
\left\langle\alpha_{\mu}\right\rangle=\left\langle\gamma_{\mu}\right\rangle=0
$$

and

$$
\left\langle\delta \alpha_{\mu} \delta \alpha_{\nu}\right\rangle=\left\langle\delta \gamma_{\mu} \delta \gamma_{\nu}\right\rangle=\delta_{\mu \nu} \epsilon^{2},
$$

where $\delta_{\mu \nu}$ is the Kronecker's delta function $(\mu, \nu=1,2,3)$ and $\epsilon$ is the isotropic noise's standard deviation. It is shown in the Appendix (Eqs. (A38)-(A40)) that for any given distribution of the noise, the averaged probabilities at optimal measurement time are approximated in the limit of large $N$ and small $\epsilon$ by:

$$
\begin{gathered}
P_{0}=1-\frac{9}{8} \pi n \sqrt{N} \epsilon^{2}+O\left(n^{2} N \epsilon^{4}\right) \\
P_{1}=\frac{\pi}{2} n \sqrt{N} \epsilon^{2}+O\left(n^{2} N \epsilon^{4}\right) \\
P_{f a r}=\frac{5}{8} \pi n \sqrt{N} \epsilon^{2}+O\left(n^{2} N \epsilon^{4}\right) .
\end{gathered}
$$


$P_{0}$ is the mean measurement probability of the marked state $|\bar{m}\rangle . P_{1}$ is the averaged probability to measure a near computational basis state which lays in the first marked state's neighborhood class (and is hence different from the marked state in a single bit only). $P_{\text {far }}$ is the averaged probability to measure a far computational basis state that differs from the marked state in more than one bit.

This result reveals some flexibility which enables us to find the marked state even when a searching error has occurred. In case of weak noise (i.e. if $\epsilon$ is small), the mean probabilities to measure near and far states are of the same order. With an approximate probability of

$\frac{P_{1}}{P_{1}+P_{\text {far }}} \approx \frac{4}{9}$, a searching error yields a near state which is different from the marked state in a single bit only. By flipping the bits of the measured near state one at a time, the marked state can be reconstructed after at most $n$ steps. Similar behavior also exists in the non-isotropic case, although the mean probabilities no longer have a compact form.

A re-scaled standard deviation magnitude:

$$
\eta=\sqrt{n \sqrt{N}} \epsilon
$$

may be considered as the parameter of the problem. The limit of $\eta \ll 1\left(\epsilon \ll \frac{1}{\sqrt{n \sqrt{N}}}\right)$ implies for weak noise in which the above approximations are valid. The limit of $\eta \gg 1\left(\epsilon \gg \frac{1}{\sqrt{n \sqrt{N}}}\right)$ is the strong noise limit where the noise completely destroys the quantum search. In this case the measured state is randomly taken from the total number of $N$ possible measured states, so that $P_{0} \rightarrow \frac{1}{N}$ and $P_{1} \rightarrow \frac{n}{N}$. In between those extremes we refer to the noise as moderate.

In the next section we present numerical results which support the analytical predictions. We evaluate the performance of the unbiased and isotropic noisy quantum search also in case of moderate noise, where the limit of weak noise is not valid anymore.

\section{THE PERFORMANCE OF THE NOISY SEARCH ALGORITHM - NUMER- ICAL RESULTS}

We first show simulation results confirming the predictions of the analytic approximation. The simulations also enable us to study the effect of larger values of $\epsilon$ (the standard deviation of the noise). These results give rise to new search strategies which we discuss in section $\mathrm{V}$.

The simulations were written in $\mathrm{C}++$ and FORTRAN, on i686 machines running Red Hat 
Linux. We applied isotropic unbiased Gaussian unitary noise by transforming the output of a uniform random number generator to be Gaussly distributed. The random Gaussian variables $\alpha_{\nu}$ and $\gamma_{\mu}(\mu=1,2,3)$ are the coefficients of the Pauli matrices as defined in Eq. (14). Note that although these coefficient are limited to the range between $-\pi$ and $\pi$, the standard deviation of the noise satisfies $\epsilon \ll 1$, thus the corrections to the Gaussian distribution are negligible. In order for the results to be statistically sound they are averaged over sufficient number of runs. The statistical error is estimated by comparing the results of different runs with identical parameters.

Fig. 1 shows the dependence of $P_{0}, P_{1}$ and $P_{f a r}$ on the standard deviation of the noise $\epsilon$, for relatively small values of $\epsilon$. The predictions of Eqs. (66), (67) and (68), are compared to the simulated results. The prediction is valid for small values of $\epsilon\left(\epsilon<0.006 \simeq 0.166 \sqrt{\frac{1}{n \sqrt{N}}}\right.$, for number of qubits in the register $n=12$ ). In the presence of noise with larger standard deviation, the next terms in the approximation are no longer negligible, and the simulated results start to deviate from the predicted ones.

Fig. 2 shows the same dependence for larger values of $\epsilon$. Results are plotted for system sizes of 8 and 15 bits. Note that a re-scaled standard deviation $\eta=\sqrt{n \sqrt{N}} \epsilon$ axis is used as predicted in Eq. (69). Indeed, this scaling makes the two graphs diverge. This further confirms the validity of the approximation.

We can roughly divide the noise deviation level into three regions:

- Weak noise deviations: $\eta \lesssim 0.166\left(\epsilon \lesssim 0.166 \sqrt{\frac{1}{n \sqrt{N}}}\right)$. This level of noise was previously discussed.

- Strong noise deviations: $\eta \gtrsim 1.4\left(\epsilon \gtrsim 1.4 \sqrt{\frac{1}{n \sqrt{N}}}\right)$. At this level of the noise Grover's algorithm should completely fail and the probability distribution among the basis states should be uniform. This means that for large $\epsilon, P_{0} \rightarrow \frac{1}{N}$, and $P_{1} \rightarrow \frac{n}{N}$ which is verified by the simulation.

- Moderate noise deviation: $0.166 \lesssim \eta \lesssim 1.4\left(0.166 \sqrt{\frac{1}{n \sqrt{N}}} \lesssim \epsilon \lesssim 1.4 \sqrt{\frac{1}{n \sqrt{N}}}\right)$. The exact behavior of Grover's algorithm under this level of noise and the specific boundaries of the moderate noise region are not predicted by the analytic approximation. However, the simulation enables us to study this case as well. As the noise increases, probability "diffuses" from the marked state to the near states and $P_{1}$ increases. Gradually, the standard deviation of the noise becomes too large, which makes the probability 
"diffuse" to all states, near and far, uniformly. In this case the vast number $(O(N))$ of far states overcomes the few $(n)$ near states, and $P_{f a r}$ approaches one.

Note that there is no noise level for which $P_{0}$ is negligible while $P_{1}$ is not. This is a results of the effect of high order terms in the approximation. Recall equations (66) and (67). For $P_{1}$ to be of the order of 1 we need $\epsilon^{2}=O\left(\frac{1}{n \sqrt{N}}\right)$, and in this case the higher order terms are $O(1)$ as well, which implies that $P_{0}$ is not necessarily negligible.

To demonstrate the flow of probability from the marked state to near and far states, we divide the computational basis vectors into classes of neighborhood to the marked state. Given the marked state vector $|\bar{m}\rangle$ we have defined its l'th order neighborhood class as the

set of $C_{l}^{n}=\frac{n !}{l !(n-l) !}$ state vectors that differ from $|\bar{m}\rangle$ in exactly $l$ bits. Let $P_{l}, l=0, \ldots, n$ denote the probability to measure any of the state vectors of the l'th neighborhood class. Fig. [3] shows the distribution of probability among the $P_{l}$ 's for different values of $\eta$. For small $\eta$, the marked state (neighborhood class of order 0) should be measured with probability 1. The remaining neighborhood classes should have zero measurement probability. For large $\eta$, the distribution of probability should be uniform among basis state vectors, which should reflect in a binomial distribution of probability among the neighborhood classes (proportional to their size). The transformation between the two extremes is determined by the two probability flow types caused by the noise: the "diffusive" and the uniform. First $P_{1}$ increases due to the "diffusive" part of the flow. Then, the middle (around $\frac{n}{2}$ ) neighborhood classes become noticeable due to the number of elements in these classes. Yet, there are cases $(0.803 \leq \eta \leq 1.069)$ where $P_{0}$ is quite small, but $P_{1}$ and $P_{2}$ are still relatively large. For even larger values of $\eta$ we have a semi-binomial distribution distorted toward the low order neighborhood classes, which eventually (for strong noise) becomes an almost pure binomial distribution.

\section{ALTERNATIVE SEARCH STRATEGIES}

One can identify two search strategies using classical or quantum search:

- Classical search until marked element is found. This strategy has an average run time of

$$
T_{\text {classical }}=\frac{N}{2}
$$


steps.

- Quantum search followed by a single classical verification step until marked element is found. This is a geometric procedure with success probability $P_{0}$ and has an average run time of

$$
T_{\text {Grover }}=\frac{\frac{\pi}{4} \sqrt{N} \tau_{q}+1}{P_{0}}
$$

where a single classical computation step is performed in one time unit and a single quantum (Grover) iteration is performed in $\tau_{q}$ time units.

The property of "diffusion" of probability from the marked state to the far states through the near ones raises the possibility of using hybrid (quantum and classical) search strategies. These strategies are based on classically searching the marked element starting from the state measured after applying Grover's algorithm, and going over the states according to the class of neighborhood to which they belong. The general hybrid strategy has a parameter $l$, and is defined as follows:

1. Run Grover's algorithm, and measure the state of the register in the computational basis at the optimal measurement time $\left(\frac{\pi}{4} \sqrt{N} \tau_{q}\right)$.

2. Repeat for $j=0 \ldots l$ : Classically search the marked state among the states of the $j$ 'th-order neighborhood class until it is found.

3. If the marked element was not found, go back to step 1.

Naturally, the average number of quantum and classical operations required in order to find the marked element depends on the noise (which controls the probabilities $P_{l}$ ), and on the value of the parameter $l$. The effectiveness of each strategy is also governed by the time it takes to perform a single quantum computation step, $\tau_{q}$ (which is the time required for a single Grover iteration).

In order to analyze the performance of each strategy we define $\left\langle T_{l}\right\rangle$ to be the average time required to complete an entire search of the marked element using the hybrid strategy with parameter $l$. We also define $\Pi_{l}$ to be the probability that the marked element is found in a search of the first $l$ neighborhood classes. Obviously,

$$
\Pi_{l}=\sum_{j=0}^{l} P_{j}
$$


Let $T_{l}$ denote the average time required to complete a single execution of steps 1 and 2 above in case that the marked element is found, and $\widetilde{T}_{l}$ denote the time required to perform a single execution of these steps in case the mark element is not found. These are given by:

$$
\widetilde{T}_{l}=\frac{\pi}{4} \sqrt{N} \tau_{q}+\sum_{j=0}^{l} C_{l}^{n}
$$

where $C_{l}^{n}$ is the binomial coefficient $C_{l}^{n}=\frac{n !}{l !(n-l) !}$, and

$$
\begin{aligned}
T_{l} & =\frac{1}{\Pi_{l}}\left[P_{0}\left(\frac{\pi}{4} \sqrt{N} \tau_{q}+1\right)+P_{1}\left(\frac{\pi}{4} \sqrt{N} \tau_{q}+1+\frac{1}{2} C_{1}^{n}\right)+P_{2}\left(\frac{\pi}{4} \sqrt{N} \tau_{q}+1+C_{1}^{n}+\frac{1}{2} C_{2}^{n}\right)+\ldots\right] \\
& =\frac{\pi}{4} \sqrt{N} \tau_{q}+1+\frac{1}{\Pi_{l}} \sum_{j=1}^{l} P_{j}\left(\sum_{i=1}^{j-1} C_{i}^{n}+\frac{1}{2} C_{j}^{n}\right) .
\end{aligned}
$$

This is the calculation of the mean time, normalized by $\Pi_{l}$.

We can now express $\left\langle T_{l}\right\rangle$ in terms of $T_{l}, \widetilde{T}_{l}$ and $\Pi_{l}$ :

$$
\begin{aligned}
\left\langle T_{l}\right\rangle & =\Pi_{l} T_{l}+\Pi_{l}\left(1-\Pi_{l}\right)\left(T_{l}+\widetilde{T}_{l}\right)+\Pi_{l}\left(1-\Pi_{l}\right)^{2}\left(T_{l}+2 \widetilde{T}_{l}\right)+\ldots \\
& =\Pi_{l} T_{l} \sum_{r=0}^{\infty}\left(1-\Pi_{l}\right)^{r}+\Pi_{l} \widetilde{T}_{l} \sum_{r=0}^{\infty} r\left(1-\Pi_{l}\right)^{r} \\
& =T_{l}+\frac{1-\Pi_{l}}{\Pi_{l}} \widetilde{T}_{l} .
\end{aligned}
$$

The average time of the optimal strategy is given by

$$
\langle T\rangle=\min _{l}\left(\left\langle T_{l}\right\rangle\right)
$$

Of course we must have $\langle T\rangle<\frac{N}{2}$. Otherwise the quantum search is completely inefficient, and the regular classical search yields better results. The best strategy parameter $l_{\text {opt }}$ is:

$$
l_{\text {opt }}=\arg \left(\min _{l}\left\langle T_{l}\right\rangle\right)
$$

where arg denotes the argument of the minimum function, i.e. the value of $l$ for which $\left\langle T_{l}\right\rangle$ is minimal.

We work under the (strict) assumption that $\tau_{q}=1$, which is that a quantum computation step requires the same amount of time as a classical computation step. Fig. [4 shows the optimal strategy averaged time $\langle T\rangle$, calculated from the data presented in Fig. 3, as a function of the noise's re-scaled standard deviation $\eta=\sqrt{n \sqrt{N}} \epsilon$ It also shows the performance of the two trivial strategies mentioned above (Eqs. 70] and [71). It is apparent 
that the required time is decreased by up to a factor of 7 . It is especially important to note that for noise deviation $\eta \gtrsim 1.4\left(\epsilon \gtrsim 1.4 \frac{1}{\sqrt{n \sqrt{N}}}\right)$ the original Grover quantum search is useless. (At the point $\eta \simeq 1.4$ the curve of the naive quantum searching time intersects with the $\frac{1}{2} N$ line which is the averaged time of a classical search). On the other hand, the optimal hybrid strategy can be used to substantially decrease the required time up to $\eta \simeq 1.9\left(\epsilon \simeq 1.9 \frac{1}{\sqrt{n \sqrt{N}}}\right)$. It is obvious that under large noise deviations the optimal hybrid strategy requires $\frac{\pi \sqrt{N}}{4} \tau_{q}+\frac{N}{2} \simeq \frac{N}{2}$ time to complete the search (this is the time required for an initial quantum search followed by a classical search of the entire search domain). As it approaches this limit $(\eta \gtrsim 1.9)$, the dependency of the time required by the hybrid strategy becomes a concave function of $\eta$. Hence, it is for this noise levels only $(1.4 \lesssim \eta \lesssim 1.9)$ that the hybrid strategy yields significant improvements. Above this level of noise, the classical search is at least as efficient (and does not require the initial quantum search).

Table $\llbracket$ shows the chosen strategy $l_{\text {opt }}$ calculated for each level of noise. It can be seen that as the noise's standard deviation increases, the chosen $l$ increases as well. This means that it is worthwhile to classically search increasingly more distant neighborhood classes for the marked element. For large $\eta, l_{\text {opt }}=20$, which means that the chosen strategy is not better than the naive classical search.

This analysis shows that the property of "diffusion" of probability from the marked state to the near states can be used to obtain a hybrid search strategy which is more efficient than the naive quantum search strategy. Note that the results above were calculated for $\tau_{q}=1$. If we make a more reasonable assumption $\tau_{q} \gg 1$ (i.e., that a single quantum computation step takes more time than a classical one), the improvement would be considerably better. In addition, the hybrid strategy enables an efficient quantum search under noise levels for which the naive quantum strategy fails.

\section{SUMMARY}

We have studied the effect of unitary noise on the performance of Grover's quantum search algorithm. This type of noise may result from tiny fluctuations and drift in the parameters of the (quantum) components performing the computation. The resulting operations are still unitary, but not precisely those assumed in the design of the algorithm. In the analysis we focused on the effect of an unbiased unitary noise in the Hadamard gate $W$. For simplicity 
we further assumed that the noise is isotropic as well. The Hadamard gate is an essential component in each iteration of the quantum search process. The gate $W$ was replaced by a noisy Hadamard gate $U$, whose parameters are distributed around those of $W$ according to an unbiased, symmetric probability distribution. The noise level was characterized by its standard deviation.

It was found that for noise levels greater than $O\left(n^{-\frac{1}{2}} N^{-\frac{1}{4}}\right)$, Grover's algorithm becomes inefficient. (Here $n$ denotes the number of qubits in the register and $N=2^{n}$ is the number of computational basis states). The nature of the flow of probability out of the marked state, which is caused by the noise was also investigated, analytically and numerically. A phenomenon of "diffusive" flow of probability to the marked state's near neighbors, that are different from the marked state in only a few bits, was observed. This feature of "diffusion" gives rise to new hybrid search strategies which are shown to improve the effectiveness of the entire search procedure, both in terms of the time required to complete the search, and in the noise level under which a quantum search is still more effective than the classical search. The use of hybrid strategies was found efficient even under noise levels for which naive re-execution of Grover's quantum search (until the marked state is found) fails.

\section{ACKNOWLEDGMENTS}

This work was supported by EU fifth framework program Grant No. IST-1999-11234.

\section{APPENDIX A: CALCULATION OF MEAN PROBABILITIES}

We are aimed to obtain an expression for the averaged probabilities to measure a state $|\bar{x}\rangle$ around the optimal measurement time in the presence of one qubit unitary noise in the Hadamard gate. We begin with Eq. (63) . As one can see $\left\langle\bar{x}\left|\hat{G}_{1}(T)\right| \overline{0}\right\rangle$ is built as sum over time index $t$ of matrix elements produced by 4 consequent operations on the initial state of zeros $|\overline{0}\rangle: \hat{Q}^{t},\left(\hat{A}_{t+1}+\hat{I}_{0} \hat{C}_{t} \hat{I}_{0}\right), \hat{Q}^{T-t}$ and $\hat{W}$ in that order. Using the matrix representations of $\hat{Q}^{t}$ and $\hat{I}_{0}$ in Eqs. (150) and (154), as well as the Hermitian noise generators presented in Eq. (55), the following operations can be expanded:

$$
\hat{Q}^{t}|\overline{0}\rangle=\cos (\omega t)|\overline{0}\rangle+\sin (\omega t) \hat{W}|\bar{m}\rangle+\vec{O}
$$




$$
\begin{aligned}
& \left(\hat{A}_{t+1}+\hat{I}_{0} \hat{C}_{t} \hat{I}_{0}\right)|\overline{0}\rangle=\left\langle\overline{0}\left|\left(\hat{A}_{t+1}+\hat{C}_{t}\right)\right| \overline{0}\right\rangle|\overline{0}\rangle+\sum_{k=0}^{n-1}\left\langle\overline{0}_{1 k}\left|\left(\hat{A}_{t+1}-\hat{C}_{t}\right)\right| \overline{0}\right\rangle\left|\overline{0}_{1 k}\right\rangle \\
& \left(\hat{A}_{t+1}+\hat{I}_{0} \hat{C}_{t} \hat{I}_{0}\right) \hat{W}|\bar{m}\rangle=\left\langle\bar{m}\left|\hat{W}\left(\hat{A}_{t+1}+\hat{C}_{t}\right) \hat{W}\right| \bar{m}\right\rangle \hat{W}|\bar{m}\rangle \\
& +\sum_{k=0}^{n-1}\left\langle\bar{m}_{1 k}\left|\hat{W}\left(\hat{A}_{t+1}+\hat{C}_{t}\right) \hat{W}\right| \bar{m}\right\rangle \hat{W}\left|\bar{m}_{1 k}\right\rangle+\vec{O}_{0} \\
& \hat{Q}^{T-t}|\overline{0}\rangle=\cos (\omega[T-t])|\overline{0}\rangle+\sin (\omega[T-t]) \hat{W}|\bar{m}\rangle+\vec{O} \\
& \hat{Q}^{T-t}\left|\overline{0}_{1 k}\right\rangle=(-1)^{T-t}\left|\overline{0}_{1 k}\right\rangle+\vec{O} \\
& \hat{Q}^{T-t} \hat{W}|\bar{m}\rangle=-\sin (\omega[T-t])|\overline{0}\rangle+\cos (\omega[T-t]) \hat{W}|\bar{m}\rangle+\vec{O} \\
& \hat{Q}^{T-t} \hat{W}\left|\bar{m}_{1 k}\right\rangle=(-1)^{T-t} \hat{W}\left|\bar{m}_{1 k}\right\rangle+\vec{O}
\end{aligned}
$$

where $\overline{0}_{1 k}$ and $\bar{m}_{1 k}$ are the binary strings which are respectively different from the initial string of zeros $\overline{0}$ and the marked string $\bar{m}$ only in their $k^{\prime}$ th bit, $\vec{O}=O\left(\frac{1}{\sqrt{N}}\right)|\overline{0}\rangle+$ $O\left(\frac{1}{\sqrt{N}}\right) \hat{W}|\bar{m}\rangle$, and $\vec{O}_{0}=O\left(\frac{n \epsilon}{\sqrt{N}}\right)|\overline{0}\rangle$ where $O(\epsilon)$ denotes the order of the noise. Then, performing consequent substitutions of Eqs. (A1)-(A7) in Eq. (63) while memorizing that $\langle\bar{x}|\hat{W}| \overline{0}\rangle=\frac{1}{\sqrt{N}}$ and $\left\langle\bar{x}|\hat{W}| \overline{0}_{1 k}\right\rangle=(-1)^{x_{k}} \frac{1}{\sqrt{N}}\left(x_{k}\right.$ is the binary value of the $k$ 'th bit in the string $\left.\bar{x}=x_{n-1}, x_{n-2}, \ldots, x_{0}\right)$ one finds that for any unmarked state $|\bar{x}\rangle$ :

$$
\begin{aligned}
\left\langle\bar{x}\left|\hat{G}_{1}(T)\right| \overline{0}\right\rangle=\frac{i}{\sqrt{N}}\left\{\sum_{t=1}^{T} \cos (\omega t) \cos (\omega[T-t])\left\langle\overline{0}\left|\left(\hat{A}_{t+1}+\hat{C}_{t}\right)\right| \overline{0}\right\rangle\right. & \\
& -\sum_{t=1}^{T} \sin (\omega t) \sin (\omega[T-t])\left\langle\bar{m}\left|\hat{W}\left(\hat{A}_{t+1}+\hat{C}_{t}\right) \hat{W}\right| \bar{m}\right\rangle \\
& \left.+\sum_{t=1}^{T} \cos (\omega t)(-1)^{T-t}\left[\sum_{k=0}^{n-1}(-1)^{x_{k}}\left\langle\overline{0}_{1 k}\left|\left(\hat{A}_{t+1}-\hat{C}_{t}\right)\right| \overline{0}\right\rangle\right]\right\} \\
+i \quad & \left\{\sum_{k=0}^{n-1}\left\langle\bar{x} \mid \bar{m}_{1 k}\right\rangle \sum_{t=1}^{T} \sin (\omega t)(-1)^{T-t}\left\langle\bar{m}_{1 k}\left|\hat{W}\left(\hat{A}_{t+1}+\hat{C}_{t}\right) \hat{W}\right| \bar{m}\right\rangle\right\} \\
& +O\left(\frac{T n \epsilon}{N}\right) \\
= & O\left(\frac{T n \epsilon}{\sqrt{N}}\right)+\sum_{k=0}^{n-1}\left\langle\bar{x} \mid \bar{m}_{1 k}\right\rangle O(T n \epsilon),
\end{aligned}
$$


where according to Eqs. (19)-(23):

$$
\begin{gathered}
\left\langle\overline{0}\left|\left(\hat{A}_{t+1}+\hat{C}_{t}\right)\right| \overline{0}\right\rangle=\sum_{k=0}^{n-1}\left\{\alpha_{0 k}(t+1)+\alpha_{3 k}(t+1)+\gamma_{0 k}(t)+\gamma_{3 k}(t)\right\} \\
\left\langle\bar{m}\left|\hat{W}\left(\hat{A}_{t+1}+\hat{C}_{t}\right) \hat{W}\right| \bar{m}\right\rangle=\sum_{k=0}^{n-1}\left\{\alpha_{0 k}(t+1)+(-1)^{m_{k}} \alpha_{3 k}(t+1)+\gamma_{0 k}(t)+(-1)^{m_{k}} \gamma_{3 k}(t)\right\} \\
\left\langle\overline{0}_{1 k}\left|\left(\hat{A}_{t+1}-\hat{C}_{t}\right)\right| \overline{0}\right\rangle=\alpha_{1 k}(t+1)+i \alpha_{2 k}(t+1)-\gamma_{1 k}(t+1)-i \gamma_{2 k}(t+1) \\
\left\langle\bar{m}_{1 k}\left|\hat{W}\left(\hat{A}_{t+1}+\hat{C}_{t}\right) \hat{W}\right| \bar{m}\right\rangle=\alpha_{3 k}(t+1)-i(-1)^{m_{k}} \alpha_{2 k}(t+1)+\gamma_{3 k}(t)-i(-1)^{m_{k}} \gamma_{2 k}(t) .
\end{gathered}
$$

We immediately observe that the order of $\left\langle\bar{x}\left|\hat{G}_{1}(T)\right| \overline{0}\right\rangle$ depends on the Hamming distance of the state $|\bar{x}\rangle$ to the marked state $|\bar{m}\rangle$. The order of "far" states, which denote binary strings different from the marked string in more than one bit is $O(\sqrt{N})$ times smaller than the order of "near" states whose binary strings are different from the marked string in exactly one bit.

According to Eq. (62), we focus on computing the mean value of $\left|\left\langle\bar{x}\left|\hat{G}_{1}(T)\right| \overline{0}\right\rangle\right|^{2}$ around the optimal measurement time. For this purpose we evaluate trigonometric sums which appear in $\left\langle\left|\left\langle\bar{x}\left|\hat{G}_{1}(T)\right| \overline{0}\right\rangle\right|^{2}\right\rangle$, assuming that $\omega T=\frac{\pi}{2}+O\left(\frac{1}{\sqrt{N}}\right)$ where $\omega \approx \frac{2}{\sqrt{N}}$.

For any small angular frequency $\omega$ and arbitrary phase $\varphi$ :

$$
\begin{aligned}
\sum_{t=1}^{T} \cos (\omega t+\varphi)(-1)^{T-t} & =\frac{\cos (\omega T+\varphi)+\cos (\omega[T+1]+\varphi)-(-1)^{T}\{\cos (\omega+\varphi)+\cos \varphi\}}{2(1+\cos \omega)} \\
& =O(1)
\end{aligned}
$$

Therefore:

$$
\begin{aligned}
& \sum_{t=1}^{T} \cos (\omega t)(-1)^{T-t}=O(1) \\
& \sum_{t=1}^{T} \sin (\omega t)(-1)^{T-t}=O(1)
\end{aligned}
$$

and:

$$
\sum_{t=1}^{T} \cos ^{2}(\omega t) \cos (\omega[T-t])(-1)^{T-t}=O(1)
$$




$$
\begin{aligned}
& \sum_{t=1}^{T} \sin (\omega t) \cos (\omega t) \cos (\omega[T-t])(-1)^{T-t}=O(1) \\
& \sum_{t=1}^{T} \sin (\omega t) \cos (\omega t) \sin (\omega[T-t])(-1)^{T-t}=O(1) .
\end{aligned}
$$

(Note that any multiplication of sines and cosines can be reduced to sums of sines and cosines).

On the other hand:

$$
\begin{aligned}
& \sum_{t=1}^{T} \sin ^{2}(\omega t)=\frac{T}{2}-\frac{\sin (2 \omega T)}{4 \tan \omega}+\frac{1}{4}\{1-\cos (2 \omega T)\}=\frac{\pi}{8} \sqrt{N}+O(1) \\
& \sum_{t=1}^{T} \cos ^{2}(\omega t)=\frac{T}{2}+\frac{\sin (2 \omega T)}{4 \tan \omega}-\frac{1}{4}\{1-\cos (2 \omega T)\}=\frac{\pi}{8} \sqrt{N}+O(1) \\
& \sum_{t=1}^{T} \cos (\omega t) \cos (\omega[T-t])=\frac{T}{2} \cos (\omega T)+\frac{\sin (\omega T)}{2 \tan \omega}=\frac{1}{4} \sqrt{N}+O(1) \\
& \sum_{t=1}^{T} \sin (\omega t) \sin (\omega[T-t])=-\frac{T}{2} \cos (\omega T)+\frac{\sin (\omega T)}{2 \tan \omega}=\frac{1}{4} \sqrt{N}+O(1) \\
& \sum_{t=1}^{T} \cos ^{2}(\omega t) \cos ^{2}(\omega[T-t])=\frac{T}{4}\left\{1+\frac{1}{2} \cos (2 \omega T)\right\}+\frac{\sin (2 \omega T)}{4}\left\{\frac{1}{\tan (2 \omega)}+\frac{1}{\tan \omega}\right\} \\
& =\frac{\pi}{32} \sqrt{N}+O(1) \\
& \sum_{t=1}^{T} \sin ^{2}(\omega t) \sin ^{2}(\omega[T-t])=\frac{T}{4}\left\{1+\frac{1}{2} \cos (2 \omega T)\right\}+\frac{\sin (2 \omega T)}{4}\left\{\frac{1}{\tan (2 \omega)}-\frac{1}{\tan \omega}\right\} \\
& =\frac{\pi}{32} \sqrt{N}+O(1) \\
& \sum_{t=1}^{T} \cos (\omega t) \cos (\omega[T-t]) \sin (\omega t) \sin (\omega[T-t])=-\frac{T}{8} \cos (2 \omega T)+\frac{\sin (2 \omega T)}{8 \tan (2 \omega)} \\
& =\frac{\pi}{32} \sqrt{N}+O(1) \text {. }
\end{aligned}
$$

We have now completed all the needed expansions for expressing the mean probability to measure a certain unmarked state $|\bar{x}\rangle$ around the optimal measurement time. By multiplying 
Eq. (A8) with its complex conjugate and averaging the value of $\left|\left\langle\bar{x}\left|\hat{G}_{1}(T)\right| \overline{0}\right\rangle\right|^{2}$ using the first and the second moments of the noise (see Eqs. (15)-(16)) while leaving the leading terms only, one obtains that the mean probability to measure an unmarked state $|\bar{x}\rangle$ around the optimal measurement time is:

$$
\begin{aligned}
p_{1} \approx & \frac{\pi}{8} \sqrt{N}\left\{\left\langle\delta \alpha_{2}{ }^{2}\right\rangle+\left\langle\delta \alpha_{3}{ }^{2}\right\rangle+\left\langle\delta \gamma_{2}{ }^{2}\right\rangle+\left\langle\delta{\gamma_{3}}^{2}\right\rangle\right\}+ \\
& \frac{1}{16}\left\{n\left[\left\langle\alpha_{3}\right\rangle+\left\langle\gamma_{3}\right\rangle\right]-f(\bar{m})\left[\left\langle\alpha_{1}\right\rangle+\left\langle\gamma_{1}\right\rangle\right]\right\}^{2}+ \\
& \left(\left\langle\alpha_{3}\right\rangle+\left\langle\gamma_{3}\right\rangle\right)^{2}+\left(\left\langle\alpha_{2}\right\rangle+\left\langle\gamma_{2}\right\rangle\right)^{2}
\end{aligned}
$$

in case that $|\bar{x}\rangle$ is a first order neighbor of the marked state $|\bar{m}\rangle$ (i.e $|\bar{x}\rangle=\left|\bar{m}_{1 k}\right\rangle$, is different from the marked state only in its $k$ 'th bit) and

$$
\begin{gathered}
p_{\text {far }} \approx \frac{1}{16} \quad\left\{n\left[\left\langle\alpha_{3}\right\rangle+\left\langle\gamma_{3}\right\rangle\right]-f(\bar{m})\left[\left\langle\alpha_{1}\right\rangle+\left\langle\gamma_{1}\right\rangle\right]\right\}^{2}+ \\
\frac{\pi}{32 \sqrt{N}}\left\{\begin{array}{l}
n\left[5\left(\left\langle\delta \alpha_{1}{ }^{2}\right\rangle+\left\langle\delta \gamma_{1}{ }^{2}\right\rangle\right)+4\left(\left\langle\delta \alpha_{2}{ }^{2}\right\rangle+\left\langle\delta{\gamma_{2}}^{2}\right\rangle\right)+\left(\left\langle\delta \alpha_{3}{ }^{2}\right\rangle+\left\langle\delta \gamma_{3}{ }^{2}\right\rangle\right)\right] \\
\left.f(\bar{m})\left[\left\langle\delta \alpha_{1} \delta \alpha_{3}\right\rangle+\left\langle\delta \gamma_{1} \delta \gamma_{3}\right\rangle\right]\right\}
\end{array}\right.
\end{gathered}
$$

in case that $|\bar{x}\rangle$ is far state (i.e. the state $|\bar{x}\rangle$ differs from the marked state $|\bar{m}\rangle$ in more than one bit). The $\alpha_{\mu}$ 's and $\gamma_{\mu}$ 's $(\mu=1,2,3)$ denote the real stochastic variables taken from any arbitrary distribution which characterize the noise, (The moments of $\alpha_{0}$ and $\gamma_{0}$ vanish because they act as a global phase). $n$ is the number of qubits in the register, $f(\bar{m})$ is the difference between the number of zeros and the number of ones that appear in the binary representation of the marked string $\bar{m}$ and $N=2^{n}$.

In the noiseless search algorithm, the $n$-qubit Hadamard transform (that consists of $n$ one qubit gates) appears twice in each iteration. These $n$ operations are expected to be implemented with similar hardware. The noise characteristics of all one qubit gates are expected to be similar but with no correlations between each other. The noisy unitary operators $\hat{U}_{t}$ and $\hat{V}_{t}$ at any Grover's iteration $t$ can therefore be considered as direct multiplications of $n$ one-qubit unitary and stochastic operators of the same statistical behavior. Thus, according to Eq. (12) the statistical properties of $\hat{c}_{k}(t)$ and $\hat{w}_{k} \hat{a}_{k}(t) \hat{w}_{k}$ are alike for any time index $t$ and qubit index $k$. Here $\hat{w}_{k}$ denotes a single-qubit operator, acting on the $k$ 'th qubit i.e.:

$$
\hat{w}_{k}=\frac{1}{\sqrt{2}}\left(\hat{\sigma}_{1 k}+\hat{\sigma}_{3 k}\right)
$$

where $\hat{\sigma}_{1 k}$ and $\hat{\sigma}_{3 k}$ are Pauli operators which act on the $k^{\prime}$ th qubit. Using the expansions of 
$\hat{a}_{k}(t)$ and $\hat{c}_{k}(t)$ by Pauli operators (see Eq. (13) ) with the aid of the identity;

$$
\left(\overrightarrow{v_{1}} \cdot \hat{\vec{\sigma}}\right)\left(\overrightarrow{v_{2}} \cdot \hat{\vec{\sigma}}\right)=\left(\overrightarrow{v_{1}} \cdot \overrightarrow{v_{2}}\right) \hat{\sigma}_{0}+i\left(\overrightarrow{v_{1}} \times \overrightarrow{v_{2}}\right) \cdot \hat{\vec{\sigma}}
$$

where $\overrightarrow{v_{1}}$ and $\overrightarrow{v_{2}}$ are 3-dimensional vectors of real numbers, $\hat{\vec{\sigma}}=\left(\hat{\sigma}_{1}, \hat{\sigma}_{2}, \hat{\sigma}_{3}\right)$ is the vector of Pauli operators, $\hat{\sigma}_{0}$ is the identity operator, and $\cdot$ and $\times$ denote scalar and vector products respectively, one obtains the following relations between statistical moments:

$$
\left\langle\gamma_{1}\right\rangle=\left\langle\alpha_{3}\right\rangle \quad\left\langle\gamma_{2}\right\rangle=-\left\langle\alpha_{2}\right\rangle \quad\left\langle\gamma_{3}\right\rangle=\left\langle\alpha_{1}\right\rangle
$$

and

$$
\begin{aligned}
& \left\langle\delta \gamma_{1}{ }^{2}\right\rangle=\left\langle\delta \alpha_{3}{ }^{2}\right\rangle \quad\left\langle\delta{\gamma_{2}}^{2}\right\rangle=\left\langle\delta \alpha_{2}{ }^{2}\right\rangle \quad\left\langle\delta \gamma_{3}{ }^{2}\right\rangle=\left\langle\delta \alpha_{1}{ }^{2}\right\rangle \\
& \left\langle\delta \gamma_{1} \delta \gamma_{2}\right\rangle=-\left\langle\delta \alpha_{2} \delta \alpha_{3}\right\rangle \quad\left\langle\delta \gamma_{1} \delta \gamma_{3}\right\rangle=\left\langle\delta \alpha_{1} \delta \alpha_{3}\right\rangle \quad\left\langle\delta \gamma_{2} \delta \gamma_{3}\right\rangle=-\left\langle\delta \alpha_{1} \delta \alpha_{2}\right\rangle
\end{aligned}
$$

Moreover, further simplification can be done, if we assume that the noise is unbiased, or alternately that the exact value of the bias of the noise is known and can be shifted in every operation of the Hadamard gate. We also assume that the physical system which realizes the quantum computer is happens to be isotropic. Thus, the statistical moments of the noise become:

$$
\left\langle\alpha_{\mu}\right\rangle=\left\langle\gamma_{\mu}\right\rangle=0
$$

and

$$
\left\langle\delta \alpha_{\mu} \delta \alpha_{\nu}\right\rangle=\left\langle\delta \gamma_{\mu} \delta \gamma_{\nu}\right\rangle=\delta_{\mu \nu} \epsilon^{2}
$$

where $\delta_{\mu \nu}$ is the Kronecker's delta function $(\mu, \nu=1,2,3)$ and $\epsilon$ is the isotropic noise's standard deviation. Then, substituting these relations in Eqs. (A26) and (A27) one gets:

$$
p_{1} \approx \frac{\pi}{2} \sqrt{N} \epsilon^{2}
$$

and

$$
p_{\text {far }} \approx \frac{5}{8} \pi \frac{n}{\sqrt{N}} \epsilon^{2}
$$

Since there are $n$ first order neighbors to the marked state $|\bar{m}\rangle$ and $N-n-1$ far states, we find that:

$$
P_{1}=n p_{1} \approx \frac{\pi}{2} n \sqrt{N} \epsilon^{2}
$$


is the mean probability to measure a first order marked state's neighbor in the optimal measurement time and

$$
P_{\text {far }} \approx N p_{f a r} \approx \frac{5}{8} \pi n \sqrt{N} \epsilon^{2}
$$

is mean measurement probability of an unmarked far state (in the optimal time as well). The mean probability to measure the marked state is given by the normalization condition:

$$
P_{0}=1-P_{1}-P_{f a r} \approx 1-\frac{9}{8} \pi n \sqrt{N} \epsilon^{2} .
$$

In order to evaluate the limits in which the above approximations are still valid, we have to estimate the order of the residual component produced by higher terms of the noise. In our calculation we have focused on the case of an unbiased noise. Therefore, only even powers of the noise standard deviation $\epsilon$ appear in the mean probabilities expansions, so the next term in the above approximations is $O\left(\epsilon^{4}\right)$. By taking those terms into account, Eq. (62) has the form:

$$
\begin{aligned}
\langle p(\bar{x}, T)\rangle \approx & \left\langle\left|\left\langle\bar{x}\left|\hat{G}_{1}(T)\right| \overline{0}\right\rangle\right|^{2}\right\rangle+\left\langle\left|\left\langle\bar{x}\left|\hat{G}_{2}(T)\right| \overline{0}\right\rangle\right|^{2}\right\rangle+ \\
& \left\langle\left(\left\langle\bar{x}\left|\hat{G}_{1}(T)\right| \overline{0}\right\rangle\left\langle\bar{x}\left|\hat{G}_{3}(T)\right| \overline{0}\right\rangle^{*}\right)\right\rangle+\left\langle\left(\left\langle\bar{x}\left|\hat{G}_{1}(T)\right| \overline{0}\right\rangle^{*}\left\langle\bar{x}\left|\hat{G}_{3}(T)\right| \overline{0}\right\rangle\right)\right\rangle
\end{aligned}
$$

where $\langle p(\bar{x}, T)\rangle$ is the mean probability to measure a certain computational basis state at time $T$ around the optimal measurement time, and $\hat{G}_{1}(T), \hat{G}_{2}(T)$ and $\hat{G}_{3}(T)$ are Grover's perturbation components of the first, second and third order respectively, as defined in Eq. (28).

An explicit calculation shows that in case that the measured state $|\bar{x}\rangle$ is a marked state first order neighbor (i.e. $|\bar{x}\rangle=\left|\bar{m}_{1 k}\right\rangle$ is different from the marked state $|\bar{m}\rangle$ in the $k^{\prime}$ 'th bit only):

$$
O\left(\left\langle\bar{x}\left|\hat{G}_{1}(T)\right| \overline{0}\right\rangle\left\langle\bar{x}\left|\hat{G}_{3}(T)\right| \overline{0}\right\rangle\right)=O\left(\left|\left\langle\bar{x}\left|\hat{G}_{2}(T)\right| \overline{0}\right\rangle\right|^{2}\right)=O\left(T^{2} n \epsilon^{4}\right)
$$

while in case that $|\bar{x}\rangle$ is far from the marked state $|\bar{m}\rangle$ :

$$
O\left(\left\langle\bar{x}\left|\hat{G}_{1}(T)\right| \overline{0}\right\rangle\left\langle\bar{x}\left|\hat{G}_{3}(T)\right| \overline{0}\right\rangle\right)=O\left(\left|\left\langle\bar{x}\left|\hat{G}_{2}(T)\right| \overline{0}\right\rangle\right|^{2}\right)=O\left(\frac{T^{2} n^{2} \epsilon^{4}}{N}\right) .
$$

Thus, due to the fact that there are $n$ first order neighbors of the marked state and $N-n-1 \approx$ $N$ far states, one finds that the mean measurement probabilities at time $T$ around the optimal measurement time (such that $O(T)=O(\sqrt{N})$ ) are given by:

$$
P_{0}=1-\frac{9}{8} \pi n \sqrt{N} \epsilon^{2}+O\left(n^{2} N \epsilon^{4}\right)
$$




$$
\begin{gathered}
P_{1}=\frac{\pi}{2} n \sqrt{N} \epsilon^{2}+O\left(n^{2} N \epsilon^{4}\right) \\
P_{f a r}=\frac{5}{8} \pi n \sqrt{N} \epsilon^{2}+O\left(n^{2} N \epsilon^{4}\right)
\end{gathered}
$$

where $P_{0}, P_{1}$ and $P_{\text {far }}$ are the mean probabilities to measure the marked state, a first order neighbor of the marked state and a far state respectively.

[1] P.W. Shor, in Proceedings of the 35th Annual Symposium on the Foundations of Computer Science, edited by S. Goldwasser (IEEE Computer Society, Los Alamitos, CA, 1994), p. 124.

[2] A. Ekert and R. Jozsa, Rev. Mod. Phys. 68, 733 (1996).

[3] L. Grover, in Proceedings of the Twenty-Eighth Annual Symposium on the Theory of Computing (ACM Press, New York, 1996), p. 212.

[4] L. Grover, Phys. Rev. Lett. 79, 325 (1997).

[5] W.H. Zurek, e-print quant-ph/0105127

[6] H. Azuma, Phys. Rev. A 65, 042311 (2002).

[7] P. Shor, Phys. Rev. A 52, 2493 (1995).

[8] P. Shor, Phys. Rev. A 54, 1098 (1996).

[9] A. Steane, Proc. R. Soc. London, Ser. A 452, 2551 (1996).

[10] A. Steane, Phys. Rev. Lett. 77, 793 (1996).

[11] E. Knill and R. Laflamme, Phys. Rev. A 55, 900 (1997).

[12] P. Zanardi, Phys. Rev. A 56, 4445 (1997).

[13] D.A. Lidar, I.L. Chuang and K.B. Whaley, Phys. Rev. Lett. 81, 2594 (1998).

[14] D. Bacon, D.A. Lidar and K.B. Whaley, Phys. Rev. A 60, 1944 (1999).

[15] J. Kempe, D. Bacon, D.A. Lidar, et al., Phys. Rev. A 63, .

[16] E. Bernstein and U. Vazirani, SIAM J. Comp. 20, .

[17] J. Preskill, Lectures notes for physics 229: Quantum Information and Computation, available from www.theory.caltech.edu/people/preskill/ph299/.

[18] M. A. Nielsen and I. L. Chuang, Quantum computation and quantum information (Cambridge University Press, Cambridge, 2000). 
[19] M. Boyer, G. Brassard, P. Hoyer and A. Tapp, in Proceedings of the fourth workshop on Physics and Computation, edited by T. Toffoli, M. Biafore and J. Leao (New England Complex Systems Institute, Boston, 1996), p. 36.

[20] C. Zalka, Phys. Rev. A 60, 2746 (1999).

[21] C.H. Bennett, E. Bernstein, G. Brassard and U. Vazirani, SIAM J. Comp. 26, 1510 (1997).

[22] E. Biham, O. Biham, D. Biron, M. Grassl and D. Lidar, Phys. Rev. A 60, 2742 (1999).

[23] L. Grover, Phys. Rev. Lett. 80, 4329 (1998).

[24] E. Biham, O. Biham, D. Biron, M. Grassl, D. Lidar and D. Shapira, Phys. Rev. A 63, 012310 (2001).

[25] C. Durr and P. Hoyer, e-print quant-ph/9607014.

[26] L. Grover, Phys. Rev. Lett. 79, 4709 (1997).

[27] L. Grover, Phys. Rev. Lett. 80, 4329 (1998).

[28] B.M. Terhal and J.A. Smolin, Phys. Rev. A 58, 1822 (1998).

[29] G. Brassard, P. Hoyer and A. Tapp, in Automata Languages and Programming, edited by K.G. Larsen, S. Skyum and G. Winskel (PUBLISHER, (Springer-Verlag, Berlin), 1998), Vol. 1443, p. 820 .

[30] N.J. Cerf, LK. Grover and C.P. Williams, Phys. Rev. A 61, 032303 (2000).

[31] R.M. Gingrich, C.P. Williams and N.J. Cerf, Phys. Rev. A 61, 052313 (2000).

[32] L. Grover, Phys. Rev. Lett. 85, 1334 (2000).

[33] A. Carlini and A. Hosoya, Phys. Lett. A. 280, 114 (2001).

[34] I.L. Chuang, N. Gershenfeld and M. Kubinec, Phys. Rev. Lett. 80, 3408 (1998).

[35] J.A. Jones, M. Mosca and R.H. Hansen, Nature 393, 344 (1998).

[36] P.G. Kwiat, J.R. Mitchel, P.D.D. Schwindt and A.G. White, J. Mod. Optics 47, 257 (2000). 
TABLE I: The list of vectors which appear in the superpositions produced by the leading perturbation components of Grover's expansion (28) with their corresponding order of the noise. The first row of the table denotes the noiseless Grover's search result at the optimal time $T_{0}$, which satisfies by definition $\hat{G}_{0}\left(T_{0}\right)|\overline{0}\rangle=|\bar{m}\rangle$. The second and third the row lists the vectors which appear in the superpositions produced by the first and the second order perturbation components $\hat{G}_{1}$ and $\hat{G}_{2}$ respectively.

\begin{tabular}{|l||l|l|}
\hline Perturbation component & Order of the noise & Vectors in the superposition \\
\hline$\hat{G}_{0}(T)|\overline{0}\rangle$ & $O\left(\epsilon^{0}\right)$ & $|\bar{m}\rangle^{a}$ \\
\hline$\hat{G}_{1}(T)|\overline{0}\rangle$ & $O\left(\epsilon^{1}\right)$ & $|\bar{m}\rangle,\left\{\left|\bar{m}_{1 k}\right\rangle\right\}_{0 \leq k \leq n-1}$ \\
& & $\hat{W}|\overline{0}\rangle,\left\{\hat{W}\left|\overline{0}_{1 k}\right\rangle\right\}_{0 \leq k \leq n-1}$ \\
\hline$\hat{G}_{2}(T)|\overline{0}\rangle$ & $O\left(\epsilon^{2}\right)$ & $|\bar{m}\rangle,\left\{\left|\bar{m}_{1 k}\right\rangle\right\}_{0 \leq k \leq n-1},\left\{\left|\bar{m}_{2 k_{1} k_{2}}\right\rangle\right\}_{0 \leq k_{1}<k_{2} \leq n-1}$ \\
& & $\hat{W}|\overline{0}\rangle,\left\{\hat{W}\left|\overline{0}_{1 k}\right\rangle\right\}_{0 \leq k \leq n-1},\left\{\hat{W}\left|\overline{0}_{2 k_{1} k_{2}}\right\rangle\right\}_{0 \leq k_{1}<k_{2} \leq n-1}$ \\
\hline$\vdots$ & $\vdots$ & $\vdots$ \\
\hline
\end{tabular}

${ }^{a}$ In case that $T$ is not the optimal measurement time the vector $\hat{W}|\overline{0}\rangle$ also appears in the list.

TABLE II: The optimal strategy $l_{\text {opt }}$ for different levels of noise deviation. The calculations are based on the data shown on Fig. [3 (number of qubits is 20). The noise re-scaled standard deviation is $\eta=\sqrt{n \sqrt{N}} \epsilon$.

\begin{tabular}{|c|c|c|c|c|c|c|c|c|c|}
\hline$\eta$ & 0.0053 & 0.138 & 0.271 & 0.404 & 0.537 & 0.670 & 0.803 & 0.936 & 1.069 \\
\hline$l_{\text {opt }}$ & 0 & 1 & 1 & 1 & 1 & 2 & 2 & 2 & 2 \\
\hline \hline$\eta$ & 1.202 & 1.335 & 1.468 & 1.607 & 1.734 & 1.867 & 2.001 & 2.133 & $\ldots$ \\
\hline$l_{\text {opt }}$ & 2 & 2 & 2 & 3 & 3 & 3 & 19 & 20 & 20 \\
\hline
\end{tabular}


Fig. 1
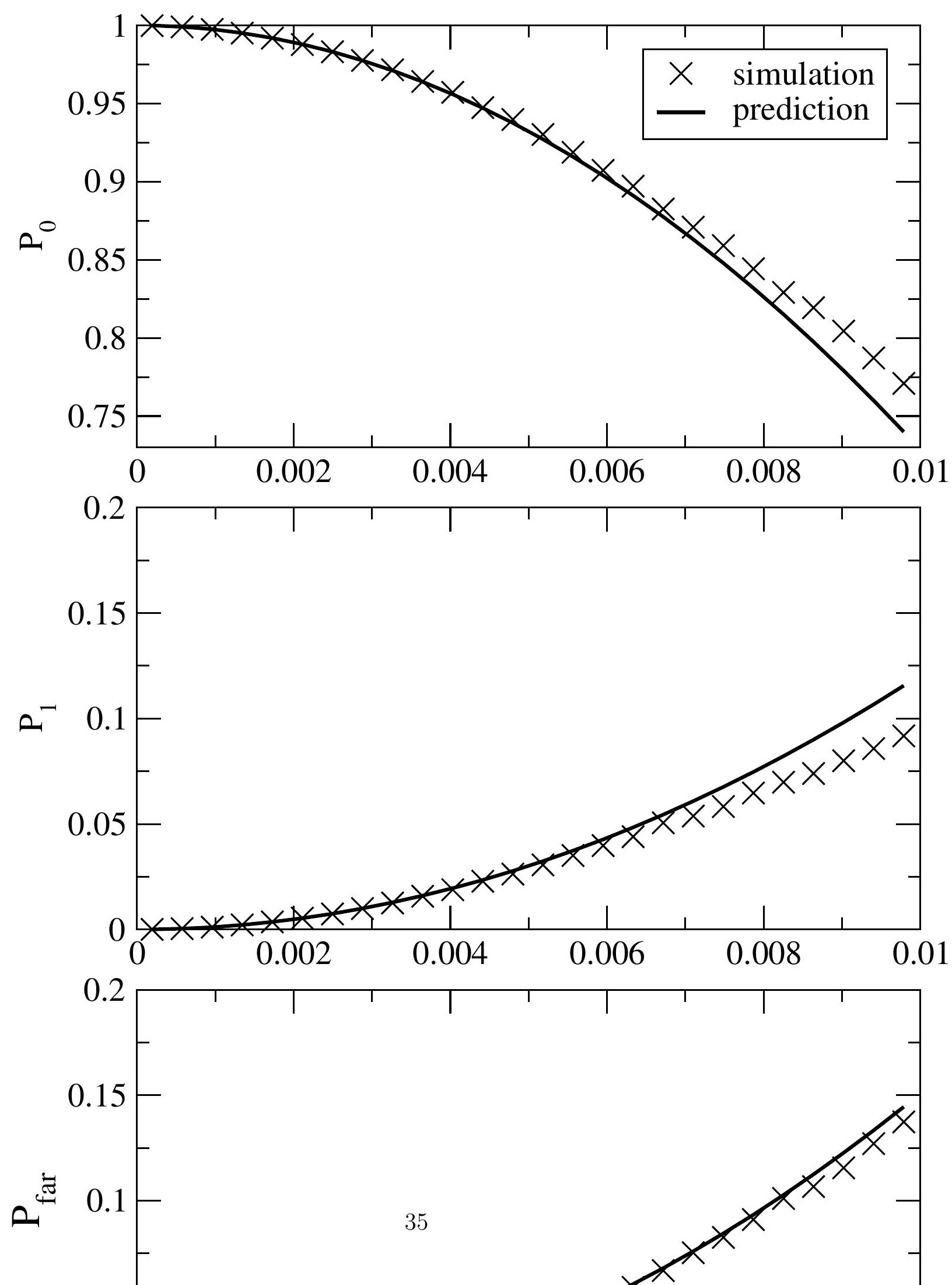

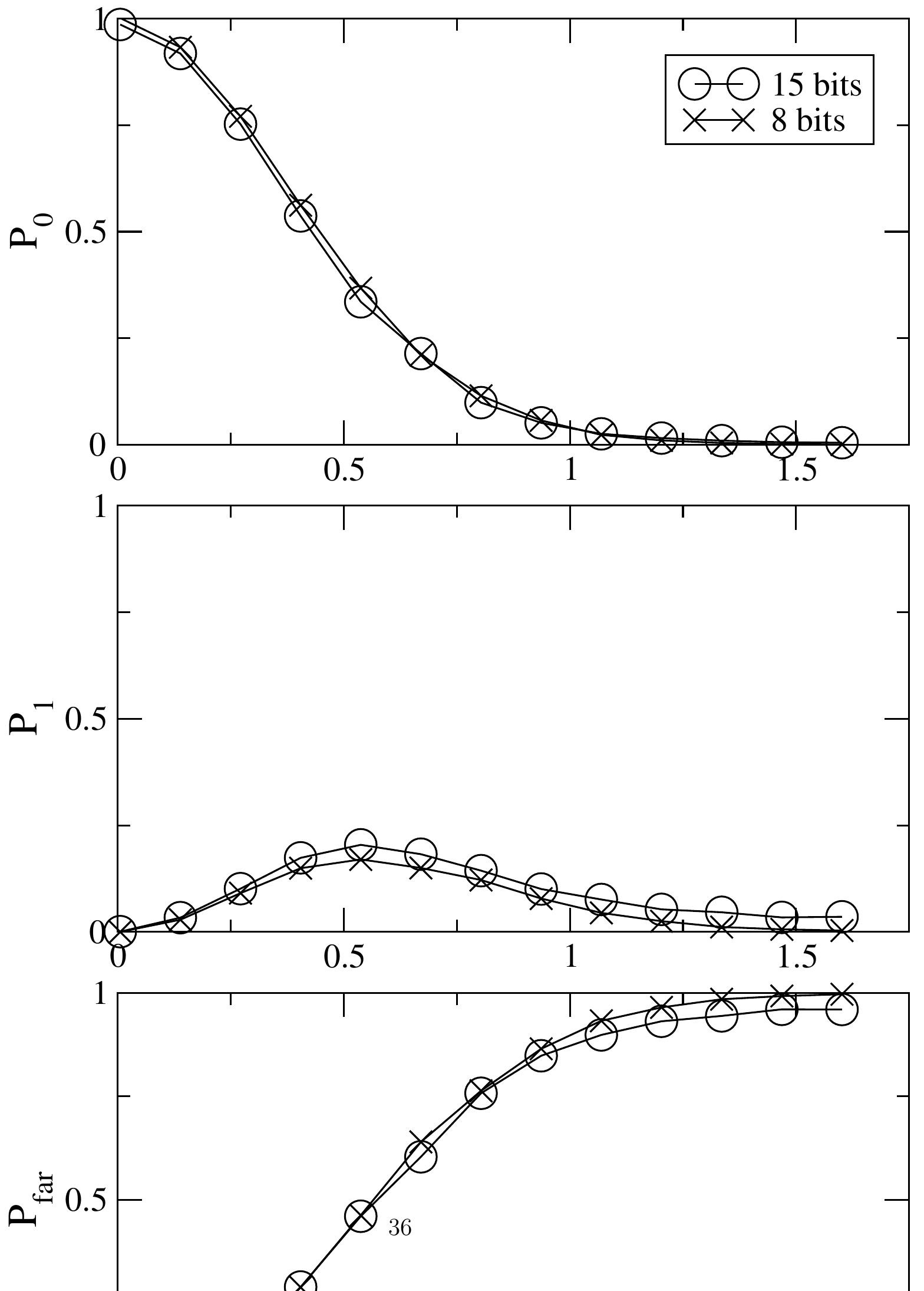

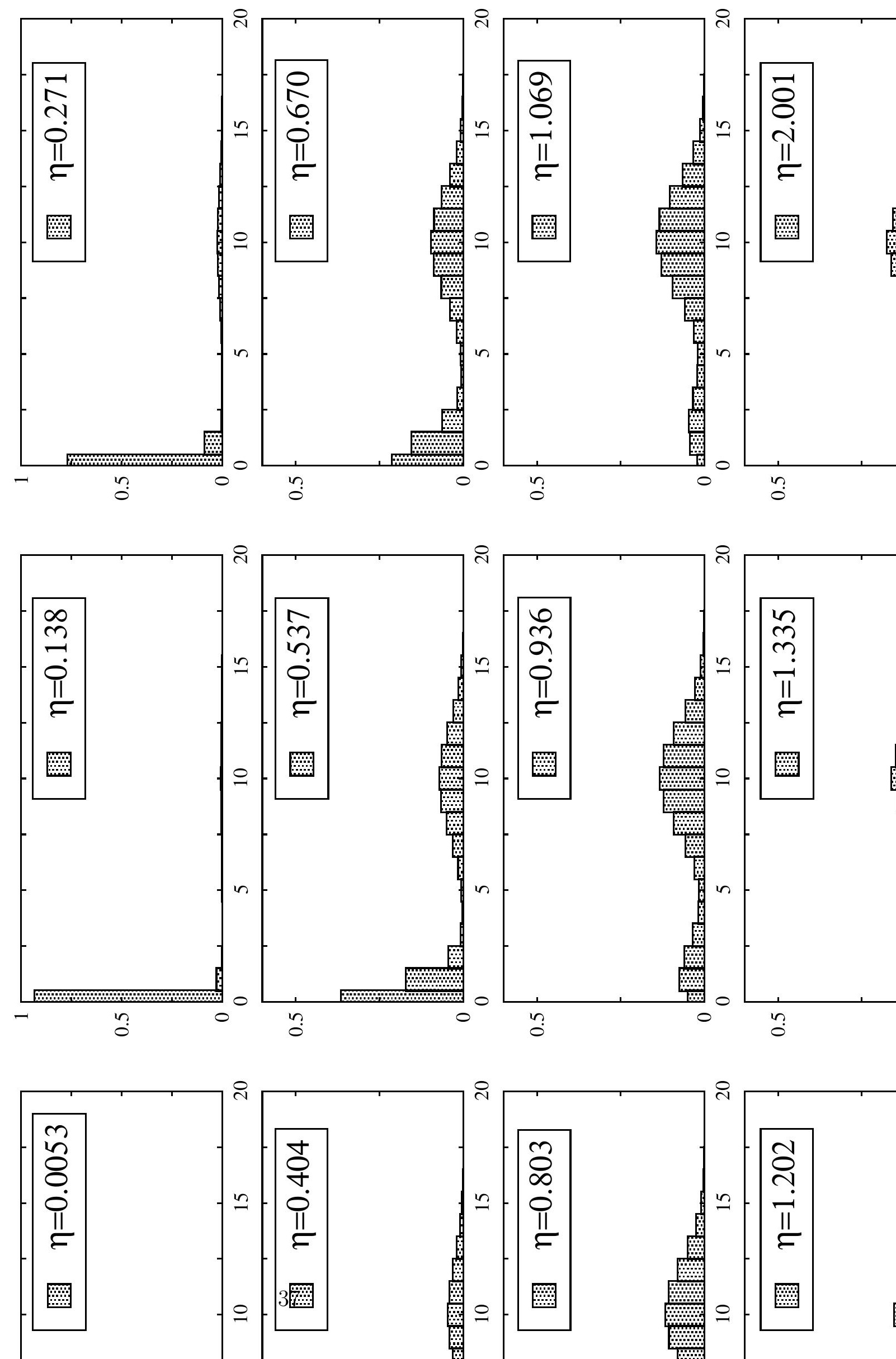


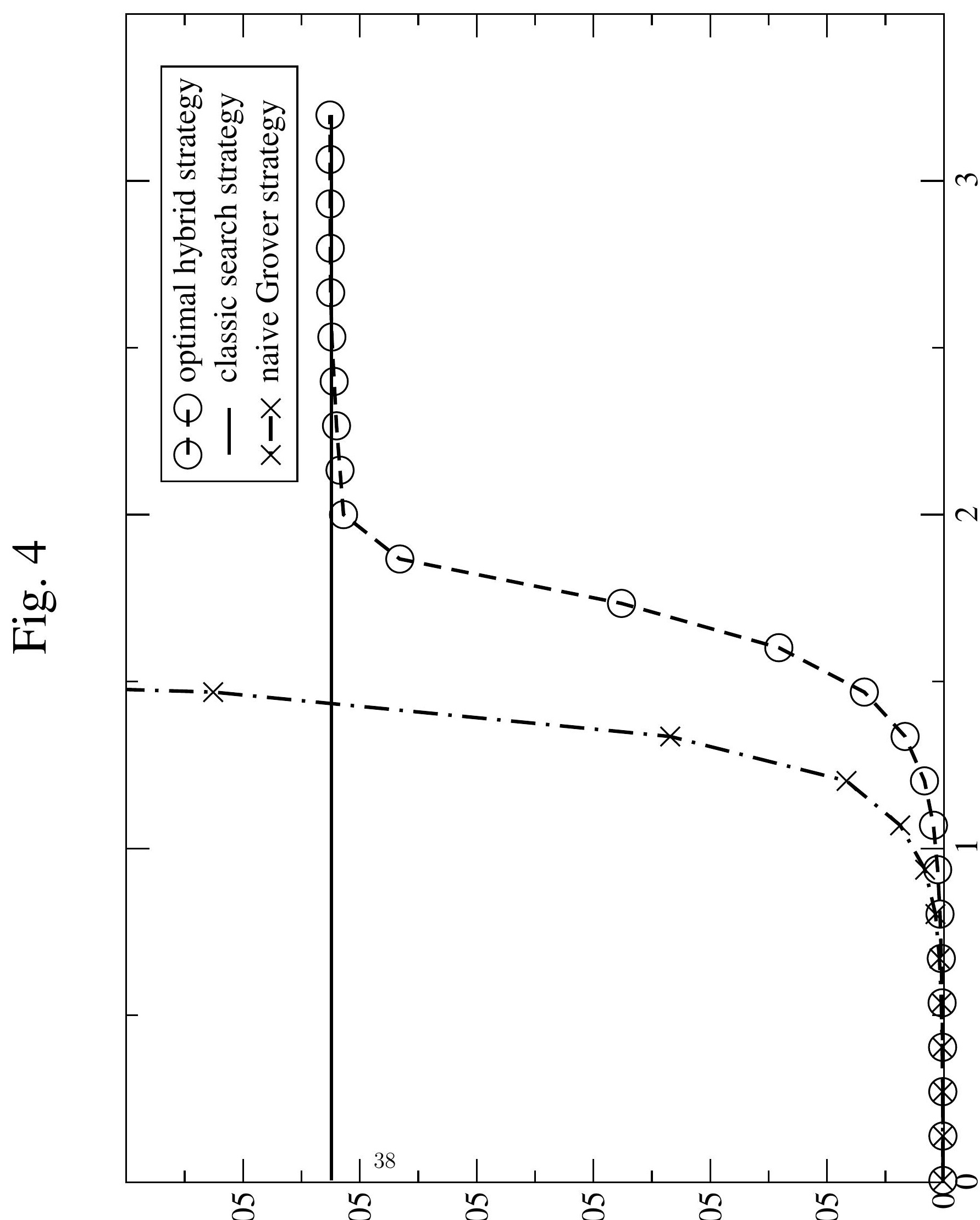

